\title{
TRIOBP-5 sculpts stereocilia rootlets and stiffens supporting cells enabling hearing
}

Tatsuya Katsuno, ${ }^{1}$ Inna A. Belyantseva, ${ }^{2}$ Alexander X. Cartagena-Rivera, ${ }^{3}$ Keisuke Ohta, ${ }^{4}$ Shawn M. Crump, ${ }^{5}$ Ronald S. Petralia, ${ }^{6}$ Kazuya Ono, ${ }^{1}$ Risa Tona, ${ }^{1,2}$ Ayesha Imtiaz, ${ }^{2}$ Atteeq Rehman, ${ }^{2}$ Hiroshi Kiyonari,, Mari Kaneko, ${ }^{7}$ Ya-Xian Wang, ${ }^{6}$ Takaya Abe, ${ }^{7}$ Makoto Ikeya, ${ }^{8}$ Cristina Fenollar-Ferrer, ${ }^{2,9}$ Gavin P. Riordan, ${ }^{2}$ Elisabeth A. Wilson, ${ }^{2}$ Tracy S. Fitzgerald, ${ }^{10}$ Kohei Segawa, ${ }^{1}$ Koichi Omori, ${ }^{1}$ Juichi Ito, ${ }^{1}$ Gregory I. Frolenkov, ${ }^{5}$ Thomas B. Friedman, ${ }^{2}$ and Shin-ichiro Kitajiri' ${ }^{1,1}$

'Department of Otolaryngology-Head and Neck Surgery, Kyoto University Graduate School of Medicine, Kyoto, Japan. 2Laboratory of Molecular Genetics, National Institute on Deafness and Other Communication Disorders, NIH, Bethesda, Maryland, USA. ${ }^{3}$ Section on Auditory Mechanics, National Institute on Deafness and Other Communication Disorders, NIH, Bethesda, Maryland, USA. ${ }^{4}$ Advanced Imaging Research Center, Kurume University School of Medicine, Kurume, Japan. ${ }^{5}$ Department of Physiology, University of Kentucky, Lexington, Kentucky, USA. ${ }^{6}$ Advanced Imaging Core, National Institute on Deafness and Other Communication Disorders, NIH, Bethesda, Maryland, USA. 'Laboratory for Animal Resources and Genetic Engineering, Riken Center for Biosystems Dynamics Research, Kobe, Japan. ${ }^{8}$ Department of Clinical Application, Center for iPS Cell Research and Application, Kyoto University, Kyoto, Japan. 'aboratory of Molecular and Cellular Neurobiology, National Institute of Mental Health, NIH, Bethesda, Maryland, USA. ${ }^{10}$ Mouse Auditory Testing Core Facility, National Institute on Deafness and Other Communication Disorders, NIH, Bethesda, Maryland, USA. "Department of Otorhinolaryngology, Shinshu University School of Medicine, Matsumoto, Japan.

TRIOBP remodels the cytoskeleton by forming unusually dense F-actin bundles and is implicated in human cancer, schizophrenia, and deafness. Mutations ablating human and mouse TRIOBP-4 and TRIOBP-5 isoforms are associated with profound deafness, as inner ear mechanosensory hair cells degenerate after stereocilia rootlets fail to develop. However, the mechanisms regulating formation of stereocilia rootlets by each TRIOBP isoform remain unknown. Using 3 new Triobp mouse models, we report that TRIOBP- 5 is essential for thickening bundles of F-actin in rootlets, establishing their mature dimensions and for stiffening supporting cells of the auditory sensory epithelium. The coiled-coil domains of this isoform are required for reinforcement and maintenance of stereocilia rootlets. A loss of TRIOBP-5 in mouse results in dysmorphic rootlets that are abnormally thin in the cuticular plate but have increased widths and lengths within stereocilia cores, and causes progressive deafness recapitulating the human phenotype. Our study extends the current understanding of TRIOBP isoform-specific functions necessary for life-long hearing, with implications for insight into other TRIOBPopathies.

Authorship note: TK and IAB contributed equally to this work.

Conflict of interest: The authors have declared that no conflict of interest exists.

Copyright: (C) 2019, American Society for Clinical Investigation.

Submitted: March 4, 2019

Accepted: May 8, 2019

Published: June 20, 2019

Reference information: /CI Insight. 2019;4(12):e128561. https://doi. org/10.1172/jici.nsight.128561.

\section{Introduction}

Variants of the human TRIOBP gene have been implicated in the pathogenesis of multiple sclerosis (1), pancreatic cancer (2), and deafness (3-7). TRIOBP encodes 3 protein size classes, TRIOBP-1 (72 kDa, NM_1024716), TRIOBP-4 (107 kDa, NM_001039155), and TRIOBP-5 (218 kDa, NM_1385579) $(5,8)$ (Figure 1A). TRIOBP was named after its first-characterized isoform, TRIOBP-1, TRIO and F-actin-binding protein-1, also known as TARA. TRIO is a regulator of cytoskeletal remodeling, cell growth, and motility that activates Rho GTPases (9-13). TRIOBP-1 has 5 predicted coiled-coil domains and a pleckstrin homology domain $(\mathrm{PH})$ (Figure 1A), binds and stabilizes actin filaments, and is required for embryonic viability $(8,14-17)$. TRIOBP-4 is predicted to be a disordered protein that binds F-actin by its R1-repeat motifs (18) and has no amino acid sequence in common with TRIOBP-1, while TRIOBP-5 includes the entire amino acid sequence of TRIOBP-4, most of the sequence of TRIOBP-1, and more (Figure 1A). Thus, TRIOBP-5 contains all of the actin-binding repeat motifs of TRIOBP-4 (18) and additional ones (14). TRIOBP-4 and TRIOBP-5 are expressed in human and mouse retina, brain, and inner ear $(5,6,8)$. 
The majority of profound deafness-associated variants of human TRIOBP are located in the large exon 6, orthologous to mouse Triobp exon 8, encoding approximately 2000 residues included in the sequence of TRIOBP-4 and TRIOBP-5 (5, 6, 8) (Figure 1A). Recently, amino acid substitutions in the sequence unique to TRIOBP-5 were found to be associated with moderate hearing loss $(4,7)$. Additionally, a genome-wide association study (GWAS) identified a noncoding variant of TRIOBP associated with a common form of adult-onset hearing loss (3). These observations underscore the medical importance of understanding TRIOBP isoform-specific functions.

Hearing loss often results from dysfunction of sensory hair cells in the cochlea $(19,20)$ (Figure 1B). Inner hair cells (IHCs) are the primary sensors of sound-induced vibrations, while outer hair cells (OHCs) are amplifiers of these vibrations (21). Sound stimuli deflect a bundle of stereocilia that project from the apical surface of both IHCs and OHCs and open mechanically gated cation channels, likely TMC1 in a complex with other proteins (22-25). Stereocilia are anchored into the apical surface of the hair cells with their rootlets embedded in the cuticular plate (26-28). In a newborn wild-type (WT) mouse (P0), rootlets are not yet visible, but each stereocilium has already developed a prominent F-actin core. Subsequently, rootlets gradually develop presumably by elongation of central actin filaments of stereocilia cores and thickening to achieve a mature shape by P16 (8, 26, 29, 30), shortly after the onset of hearing (31-33). A mature rootlet has the appearance of a double-pointed needle centrally positioned at the pivot point of a WT stereocilium, extending into its F-actin core about a third to half the length of a stereocilium (referred here as the upper half of a rootlet) and approximately the same distance into the actin meshwork of the cuticular plate (the lower half of a rootlet) (26). Actin filaments within the rootlets are tightly packed with no noticeable space between them, an observation that we recapitulated in vitro using F-actin bundled by purified TRIOBP-4 $(8,18)$.

We previously engineered a knockout mouse by removing exon 8 of Triobp (Triobp ${ }^{4 E \times 8 / \Delta E \times 8}$ ), thus simultaneously ablating TRIOBP-4 and TRIOBP-5 isoforms (Figure 1C), while leaving the expression of TRIOBP-1 intact (8). A Triobp ${ }^{4 E \times / \triangle E x 8}$ mouse never develops stereocilia rootlets and is congenitally, profoundly deaf (8). Stereocilia lacking rootlets initially have a normal appearance and are capable of mechanotransduction but are fragile (8). Shortly after the onset of hearing at P16, these floppy stereocilia lacking rootlets fuse together and rapidly degenerate, followed by the death of hair cells (8), presumably the pathogenic process accounting for human profound deafness due to mutations of $\operatorname{TRIOBP}(5,6)$. However, the potential differences in function of TRIOBP-4 and TRIOBP-5 as well as the mechanisms regulating the overall architecture of stereocilia rootlets including their width, length, and shape are unknown.

Here, we determined the specific roles of TRIOBP-4 and TRIOBP-5 in the formation of F-actin bundles of stereocilia rootlets using 2 isoform-specific knockout mouse models, measurements of hair cell mechanical properties using PeakForce Tapping atomic force microscopy and water-jet stimulations, live cell nanoscale pull-down protein-protein interaction assays, as well as $3 \mathrm{D}$ reconstructions of rootlets from electron microscopy images. We show that rootlets have 2 separate compartments with different compositions of TRIOBP isoforms. Although TRIOBP-4 alone can initiate rootlet formation, a TRIOBP-5 deficiency results in rootlet dysmorphology and progressive deafness in 2 independent mouse models, mimicking the human phenotype. We also show that WT TRIOBP-5 is essential for the precise dimensions and mechanical resilience of rootlets. Moreover, TRIOBP-5 contributes to the apical stiffness of flanking supporting cells for life-long hearing.

\section{Results}

TRIOBP-4 and TRIOBP-5 localize to different rootlet compartments. Immunostaining of WT mouse auditory epithelia with TRIOBP-5-specific antiserum (Figure 1A) revealed TRIOBP-5 localization restricted to the lower half of rootlets, immediately below the apical surface of a hair cell within the cuticular plate (Figure 2A). In contrast, the TRIOBP-4/5 antibody that detects both TRIOBP-4 and TRIOBP-5 isoforms (Figure 1A) stained stereocilia and the entire length of rootlets with a prominent signal present in the F-actin stereocilia core, as previously reported (8) (Figure 2D, inset). This observation indicates that localization of TRIOBP-4 is different from TRIOBP-5. However, an antibody that specifically detects only TRIOBP-4 could not be developed, as TRIOBP-4 and TRIOBP-5 have identical amino-terminal sequences. To circumvent this problem, we developed an R26-EGFP-Triobp-4 transgenic reporter mouse (Supplemental Figure 1; supplemental material available online with this article; https://doi.org/10.1172/jci.insight.128561DS1). EGFP-TRIOBP-4 was predominantly localized in the upper half of rootlets within stereocilia cores of homozygous R26-EGFP-Triobp-4 reporter mice, while a weak EGFP-TRIOBP-4 signal was observed 
A

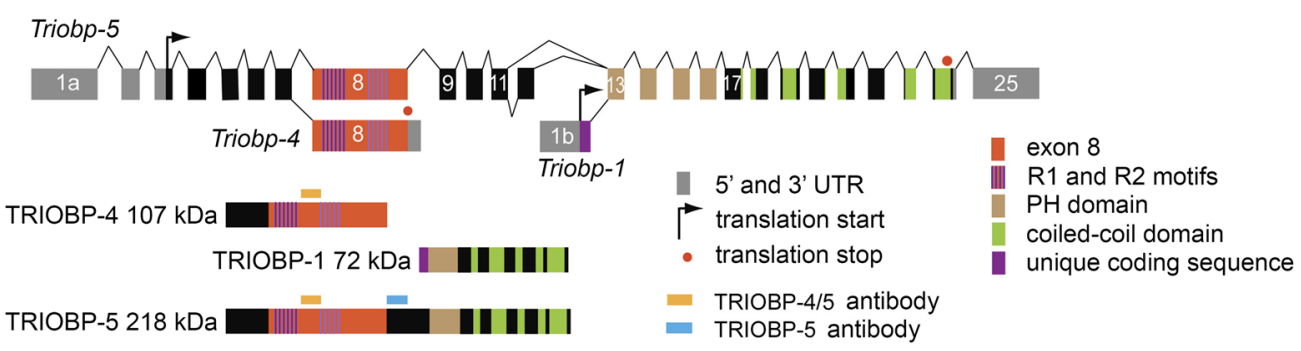

B

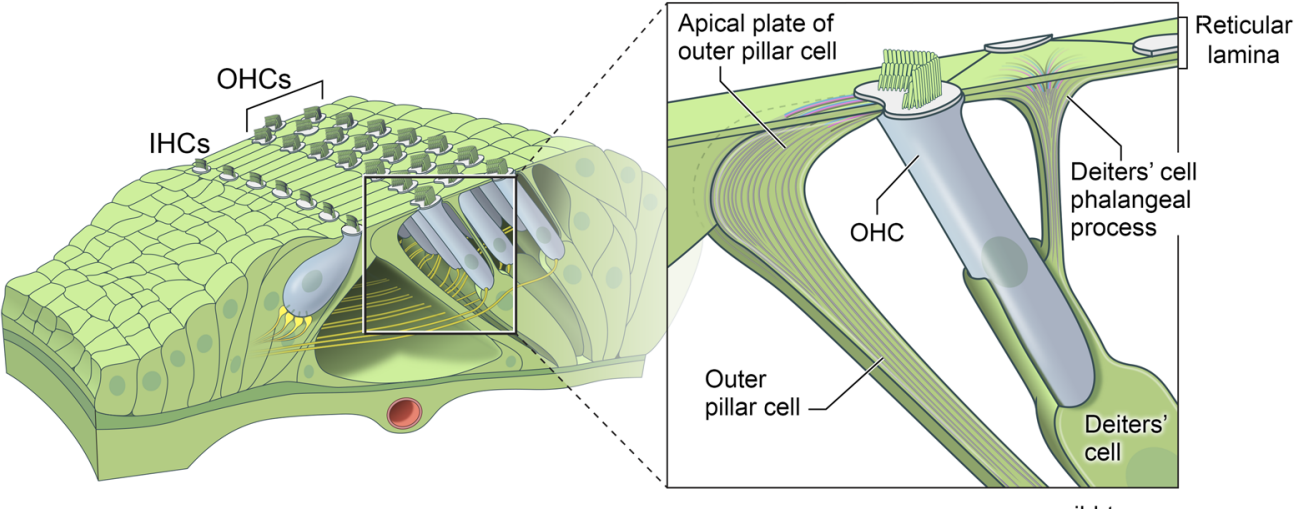

C 1. Triobp ${ }^{\triangle E x \cdot-10}$ (deletion of exons 9 and 10 and insertion of LacZ)

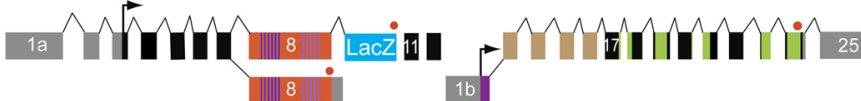

wild-type

Triobp ${ }^{\Delta E \times 8}$ (exon 8 sequence replaced with LacZ)

\section{1a hinith Laci}

mintinturint $1 \mathrm{~b}$

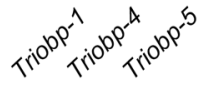

yes yes no

3. Triobp $^{\text {ҮHB226 }}$ (gene trap YHB226 inserted in exon 17)

\section{1a}

4. Triobp ${ }^{\triangle E \times 8 / Y H B 226}$ (compound heterozygote combining alleles 2 and 3 in trans) no yes no

yes yes no

Figure 1. Triobp gene structure, transcripts, protein isoforms, and mutant alleles. (A) Alternative transcripts of mouse Triobp, the corresponding encoded protein isoforms, and their predicted domains. Locations of the epitopes for 2 antibodies (TRIOBP-4/5 and TRIOBP-5) are depicted with orange and light-blue rectangles, respectively. (B) Drawing of an organ of Corti sensory epithelium segment showing 1 row of inner (IHC) and 3 rows of outer (OHC) hair cells. Inset shows Deiters' and outer pillar cells supporting an OHC. (C) Mutations of Triobp used to generate 2 genetically different TRIOBP-5-deficient mouse models. Triobp ${ }^{4 \times \times 9-10 / 4 E \times 9-10}$ has a LacZ cassette replacing exons 9 and 10 of Triobp-5 (schematic 1). The Triobp ${ }^{4 E \times 8}$ allele has a LacZ cassette replacing exon 8 (schematic 2 ) and the Triobp ${ }^{\text {YHBz26 }}$ allele has a trap cassette with a LacZ insertion in exon 17 (schematic 3 ). The combination in trans of these 2 Triobp mutations is a compound heterozygote designated Triobp ${ }^{4 \times \times 8 / Y H B 226}$. Expression of wild-type Triobp isoforms 1,4 , and 5 is indicated to the right of the schematics for each genotype.

throughout the hair cell cuticular plate, likely masking a weak EGFP-TRIOBP signal in the lower half of stereocilia rootlets (Figure 2, A and B, and Supplemental Figure 2). The EGFP-TRIOBP-4 signal was very similar to the immunofluorescence detected in the WT using the TRIOBP-4/5 antibody (Figure 2D), demonstrating that the predominant localization of endogenous TRIOBP-4 is in the upper half of rootlets embedded in stereocilia cores (Figure 2, A, B, and D). In contrast, immunostaining of endogenous TRIOBP-5 in the R26-EGFP-Triobp-4 reporter mouse confirmed the restricted localization of TRIOBP-5 in the lower half of a rootlet within the cuticular plate (Figure $2 \mathrm{~A}$ ).

A filamentous pattern of EGFP-TRIOBP-4 fluorescence was also detected in the upper portions of the ascending phalangeal processes of Deiters' supporting cells that flank hair cells (Figure 1B and Figure 2C, 


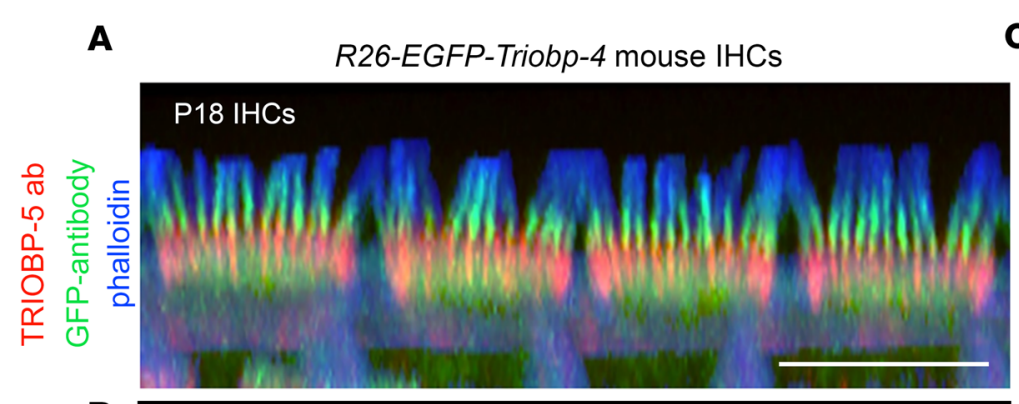

C R26-EGFP-Triobp-4 mouse organ of Corti
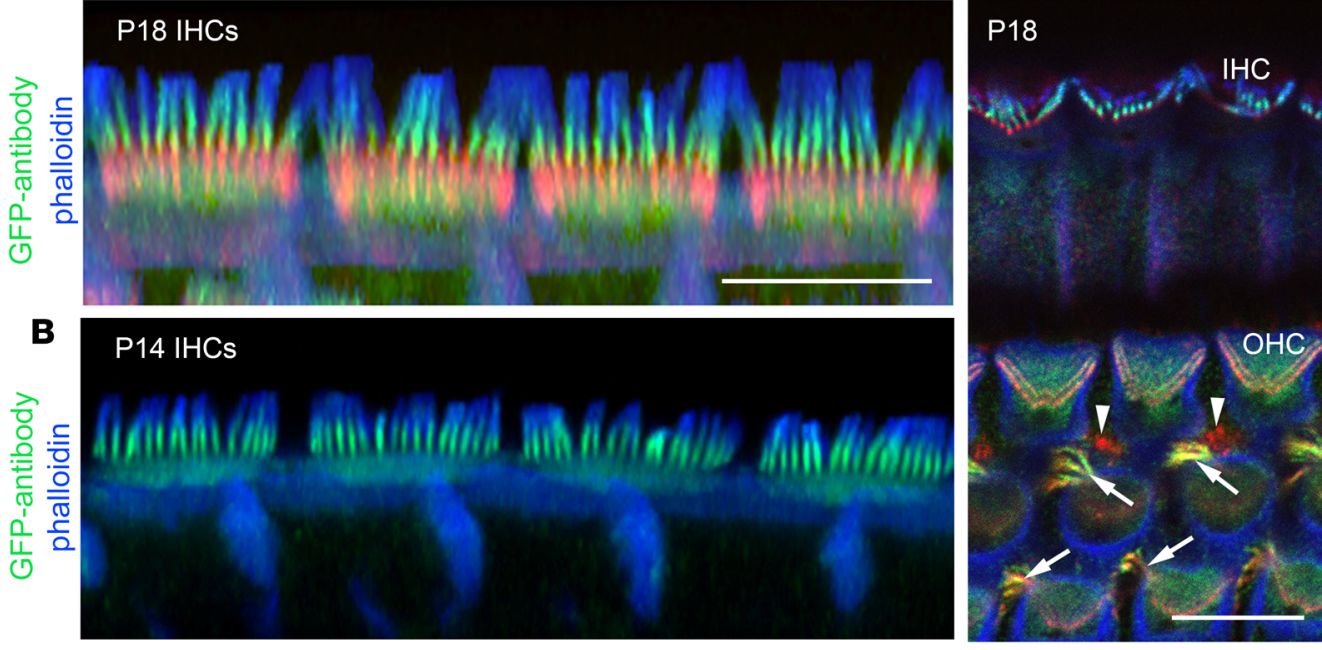

D

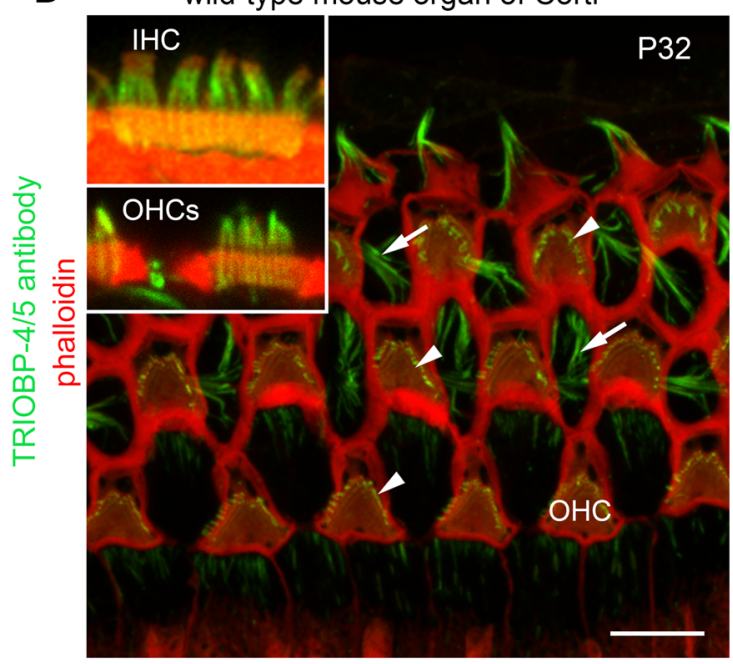

E
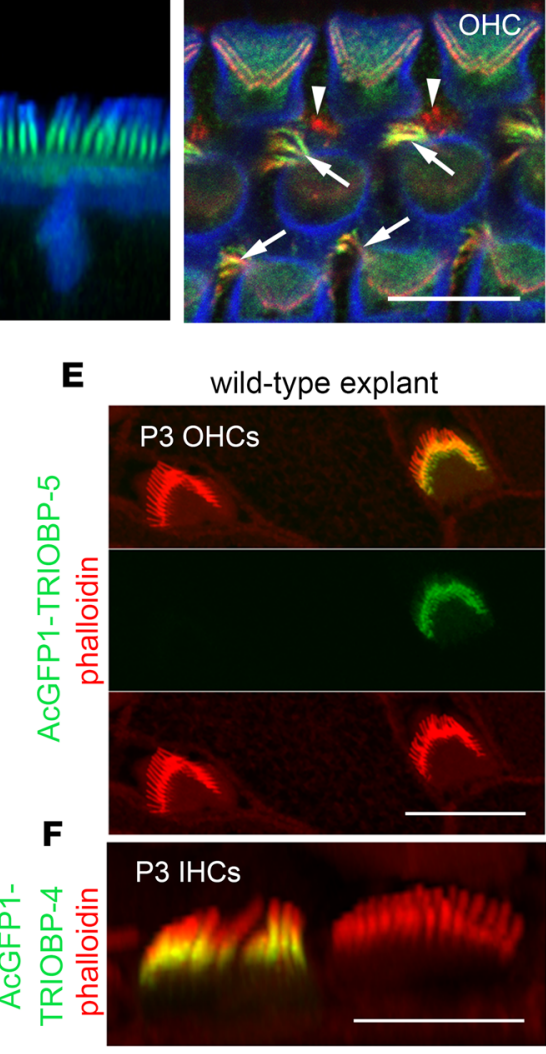

Figure 2. Differential localization of TRIOBP isoforms in stereocilia rootlets revealed in the R26-ECFP-Triobp-4 transgenic reporter mouse. (A) TRIOBP-5specific antibody signal (red) observed predominantly in the rootlet segment inside the cuticular plate. See single channel images in Supplemental Figure 2. (B) Anti-GFP antibody recognized EGFP-TRIOBP-4 in the stereocilia rootlet segment that resides above the cuticular plate within the stereocilia core (green). A diffuse EGFP signal is present in the cuticular plate (green). (C) In Deiters' cells located between OHCs, both TRIOBP-4 and TRIOBP-5 are present in F-actin bundles of obliquely ascending processes (arrows). Outer pillar cells show predominantly a TRIOBP-5 signal (red, arrowheads) close to junctions with OHCs.

(D) P32 wild-type mouse (C57BL/6J) organ of Corti stained with TRIOBP-4/5 antibody. The inset shows representative IHC, in which TRIOBP-4/5 immunoreactivity (green) is prominent in stereocilia above the cuticular plate and a weaker signal is present in the rootlet segment within the cuticular plate. TRIOBP-4/5 immunoreactivity is also present in stereocilia of $\mathrm{OHCs}$ (inset and arrowheads) and in Deiters' cells (arrows). (E and F) Helios gene gun-mediated transfections of P3 wild-type organ of Corti explants with plasmids expressing either AcCFP1-TRIOBP-5 (E) or AcCFP1-TRIOBP-4 CDNA (F) reveal targeting of AcGFP1-TRIOBP-5 (E) to stereocilia rootlets (green) within the cuticular plate. (F) AcCFP1-TRIOBP-4 predominantly targets the upper half of the rootlets within the stereocilia core, recapitulating the endogenous differential localization of TRIOBP-4 and TRIOBP-5. A barely detectable signal of AcGFP1-TRIOBP-4 was found in the lower half of rootlets within the cuticular plate. F-actin is visualized by blue phalloidin- 405 in A-C and rhodamine-phalloidin (red) staining in D-F. All images are maximum intensity projections of confocal Z-stacks or their subsets (inserts). Scale bars: $5 \mu \mathrm{m}$ (all panels).

arrows). Filamentous structures near the junctions with OHCs in the heads of outer pillar supporting cells (Figure 2C, arrowheads) were also highlighted by TRIOBP-5 antibody staining (Figure 2C, red). Supporting cells are thought to be important for mechanical stiffness of the cochlear partition that shapes sound-induced intracochlear vibrations $(34,35)$.

Next, AcGFP1-TRIOBP-4 and AcGFP1-TRIOBP-5 were introduced into WT hair cells of P3 mouse organ of Corti explants by biolistic gene-gun transfections (36). We observed isoform-specific targeting of exogenous AcGFP1-TRIOBP-5 (Figure 2E) and AcGFP1-TRIOBP-4 (Figure 2F) to the same subcellular 
locations that were revealed using antisera and the R26-EGFP-TRIOBP-4 reporter mouse, confirming the differential localization of these isoforms within stereocilia. These data indicate that the upper half of a rootlet within the F-actin core of a stereocilium and its lower half within the cuticular plate have different TRIOBP isoform compositions, even though the entire length of rodent auditory hair cell rootlets appear as a single uniform electron-dense F-actin structure when examined by transmission electron microscopy (TEM) (26).

TRIOBP-5-deficient mice exhibit progressive deafness. The hearing phenotype of mice deficient only for the TRIOBP-5 isoform is unknown. We used 2 different genetic strategies to engineer new mouse models each deficient only for TRIOBP-5. One TRIOBP-5-deficient mouse model was engineered by deleting exons 9 and 10 (Tri$o b p^{4 E x 9-19}$ ) unique to TRIOBP-5 and replacing them with a LacZ reporter cassette followed by a translation stop codon (Figure 1C and Supplemental Figure 1). Southern blot analyses confirmed the deletion of exons 9 and 10 (Supplemental Figure 1). RT-PCR detected no Triobp-5-specific mRNA from the inner ear of homozygous mutant mice and no alteration in expression of Triobp- 1 and Triobp- 4 isoforms (Supplemental Figure 1). Immunostaining with anti-TRIOBP-5 antibody detected no TRIOBP-5 protein in stereocilia rootlets of homozygous Triobp-5 $5^{4 E x-10 / \triangle E x 9-10}$ mice (Supplemental Figure 1), validating the specificity of this antiserum.

In a Triobp $p^{4 E x 9-10 /+}$ heterozygous mouse, $\beta$-galactosidase activity was detected in spiral ganglion neurons and in the sensory epithelium of the organ of Corti (Supplemental Figure 1). This pattern is similar to the $\beta$-galactosidase expression we reported for a heterozygote Triobp ${ }^{4 E \times 8 /+}$ mouse (8) and indicates that the mRNAs for the Triobp- 4 and Triobp-5 isoforms are each expressed in the same inner ear cell types.

A second TRIOBP-5-deficient mouse (Triobp ${ }^{4 E \times 8 / Y H B 226}$ ) was generated by crossing a homozygous Tri$o b p^{4 E \times 8 / \triangle E \times 8}$ mouse with a heterozygous Triobp ${ }^{\text {YHB226/+ }}$ mouse (Figure 1C). The Triobp ${ }^{\text {YHB226 }}$ allele has a gene trap inserted in exon 17, which disrupts expression of TRIOBP-5 and TRIOBP-1. Homozygosity for Tri$o b p^{\text {YHB226 }}$ results in embryonic lethality due to a lack of TRIOBP-1 (8). Compound heterozygous Triobp ${ }^{\unlhd E \times x /}$ YHB226 mice express TRIOBP-1 from the Triobp ${ }^{4 E x 8}$ allele, while the Triobp ${ }^{\text {YHB226 }}$ allele allows transcription of WT Triobp-4 (Figure 1, A and C). Both Triobp ${ }^{4 E x 9-10 / \triangle E x 9-10}$ mice and TRIOBP-5-deficient compound heterozygous mice were obtained at the expected Mendelian ratio and macroscopically were indistinguishable from their WT littermates. To evaluate the hearing of both TRIOBP-5-deficient mouse models, auditory brainstem responses (ABRs) were recorded (Figure 3). At 4 and 8 weeks of age, Triobp ${ }^{4 E \times 8 / Y H B 226}$ mice have residual hearing at 8 and $16 \mathrm{kHz}$. By 12 weeks of age hearing loss was severe to profound at all frequencies tested (Figure 3A). Four-week-old Triobp ${ }^{4 E x 9-10 / \triangle E x 9-10}$ mice also had residual hearing at 8 and $16 \mathrm{kHz}$ that progressed to severe to profound hearing loss by 8 weeks of age (Figure 3B), indicating a more rapid progression of hearing loss as compared with Triobp ${ }^{4 E \times 8 / Y H B 226}$. These deaf mice have no obvious vestibular abnormalities such as circling or head bobbing.

To evaluate OHC function, we recorded distortion product otoacoustic emissions (DPOAEs) in Triob$p^{4 E \times 8 / Y H B 226}$ and Triobp $p^{4 E x 9-10 / \triangle E x 9-10}$ mice and their littermate controls. DPOAEs measure cochlear amplification that requires functional OHCs (37). DPOAEs were not detected in Triobp $p^{4 E x 9-10 / \triangle E x 9-10}$ or Triobp ${ }^{4 E x 8 / Y H B 226}$ mice by 8 weeks of age. At 4 weeks of age, DPOAEs were absent in 7 of the 16 Triobp ${ }^{4 E \times 8 / Y H B 226}$ mice, and the remaining 9 only had low-level DPOAEs within the $8-20 \mathrm{kHz}$ range (Supplemental Figure 3). DPOAEs were absent in 7 Triobp $^{4 E x 9-10 / \triangle E x 9-10}$ mice at 4 weeks of age, with the exception of one mouse with very low DPOAE levels restricted to the $8-11.2 \mathrm{kHz}$ range (Supplemental Figure 3). These data indicate a loss of OHC function in both TRIOBP-5-deficient mouse models, which is consistent with progressive degeneration of hair cells described below.

Degeneration of hair cell stereocilia in mice deficient for TRIOBP-5. Scanning electron microscopy (SEM) revealed a structural explanation for hearing loss in the organs of Corti from TRIOBP-5-deficient mice (Triobp ${ }^{\Delta E x 9-10 / \Delta E x 9-10}$ ) and littermate normal hearing controls at P7, P14, P30, and P40. Initially, stereocilia bundles of TRIOBP-5-deficient mice are indistinguishable from controls (Supplemental Figure 4). After P14, hair bundles of TRIOBP-5-deficient mice become disorganized, and many stereocilia of IHCs are fused with one another. By P40, we observed a loss of some stereocilia from OHC hair bundles, predominantly from the longest stereocilia row, perhaps due to their detachment at fragile pivot points near the apical surface of a hair cell (Figure 3D and Supplemental Figure 4). A similar but slower progressive degeneration of the stereocilia bundles was observed in the Triobp ${ }^{4 E x 8 / \text { YHB226 }}$ TRIOBP-5-deficient mouse (Figure $3 \mathrm{C}$ and Supplemental Figure 5), consistent with a milder progressive loss of hearing.

TRIOBP-5-deficient mice have dysmorphic rootlets. We hypothesized that in the absence of TRIOBP-5, TRIOBP-4 alone would be capable of forming a rootlet-like structure because F-actin and purified TRIOBP-4 in vitro can form tight actin bundles reminiscent of rootlets (8). To test this hypothesis, ultrastruc- 
A

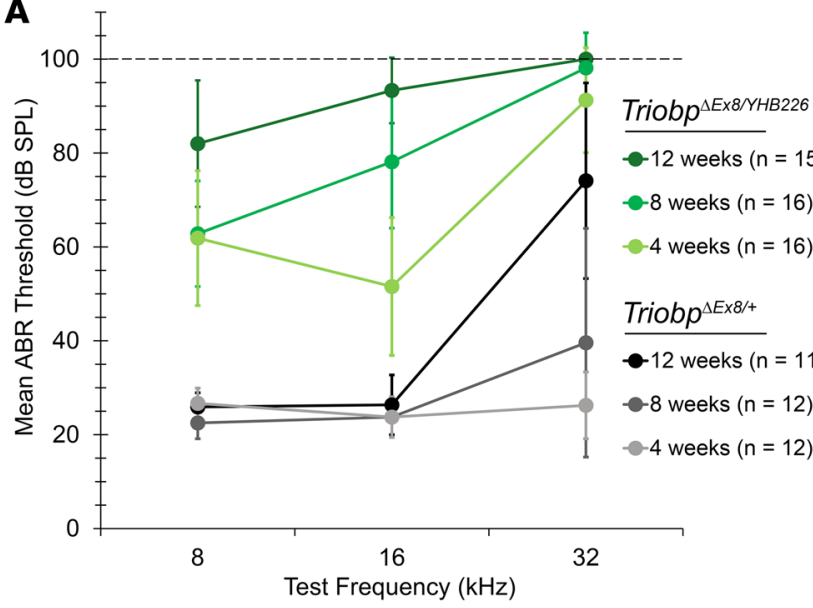

B

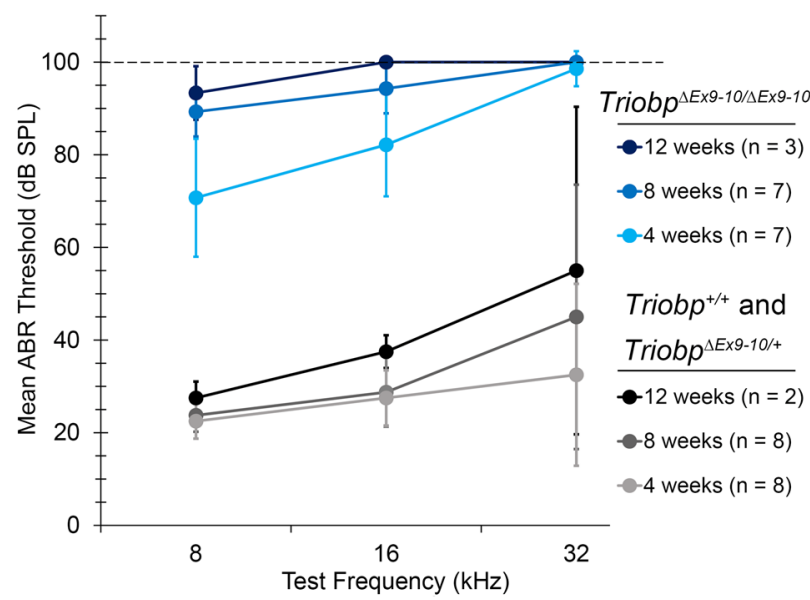

\section{C}
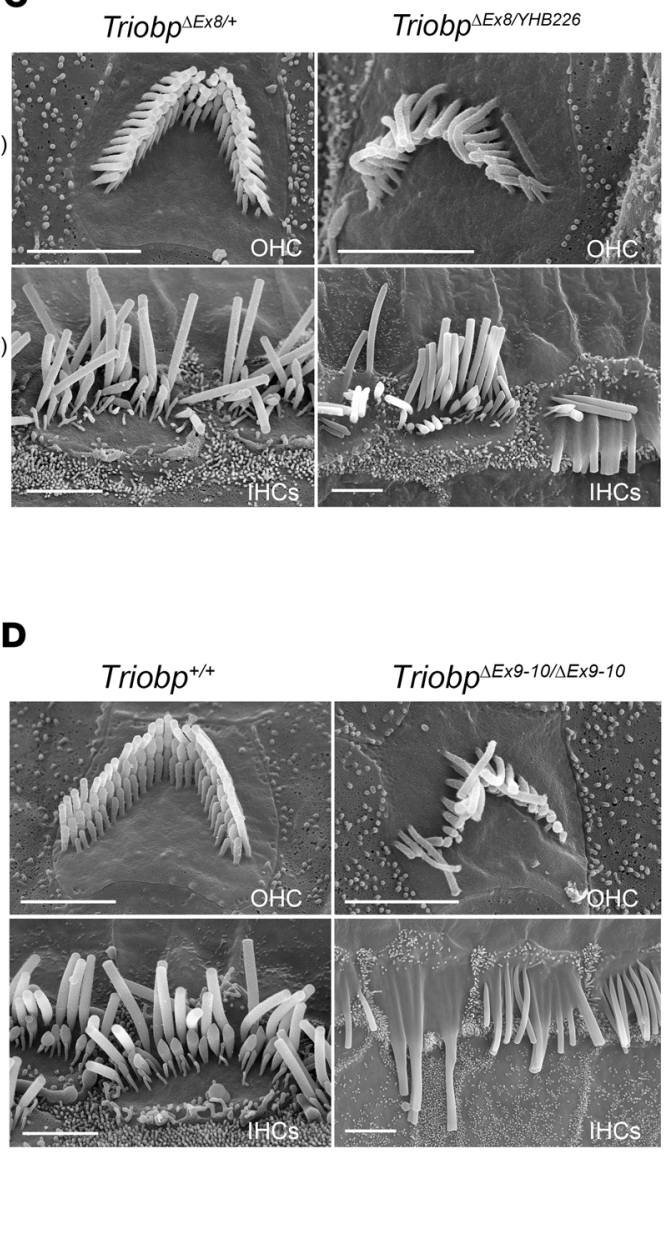

Triobp $\Delta E \times 9-10 / \Delta E \times 9-10$

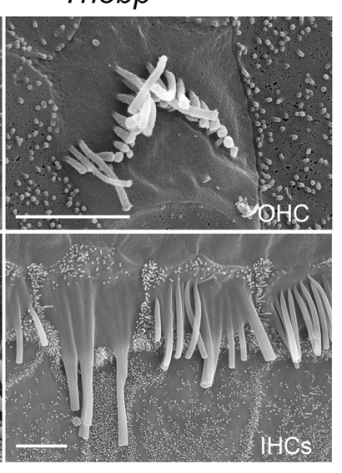

Figure 3. Progressive hearing loss in TRIOBP-5-deficient mice. (A) Average auditory brainstem response (ABR) thresholds in Triobp ${ }^{4 E \times 8 /+}$ heterozygous control mice (gray/black) and Triobp ${ }^{4 \times \times 8 / Y H B 226}$ compound heterozygous TRIOBP-5-deficient mice (shades of green). (B) Average ABR thresholds in Triobp ${ }^{+/+}$ (wild-type) and Triobp $p^{4 \mathrm{Exg}-10 /+}$ heterozygous controls (gray/black) and in Triobp ${ }^{4 \times \times 9-10 / 1 E \times 9-10}$ mice (shades of blue). ABRs were measured at 4,8 , and 12 weeks postnatally at frequencies of 8,16 , and $32 \mathrm{kHz}$. When no response was detected at a maximum stimulus level of $90 \mathrm{~dB}$ SPL, the threshold was assigned as $100 \mathrm{~dB}$ SPL (dashed line in A and B). Error bars indicate SD. (C) SEM images of P43 mouse IHC and OHC stereocilia bundles from normal-hearing Triobp ${ }^{4 \mathrm{E} \times 8 /+}$ heterozygotes (left) and from deaf Triobp ${ }^{4 \times \times 8 / Y H B 226}$ (right) compound heterozygotes. (D) SEM images of mouse P35 stereocilia bundles of wild-type and Triobp ${ }^{\Delta E \times g-10 / \triangle E x g-10} \mathrm{OHCs}$ and IHCs. Note fusion of stereocilia of mutant IHC and loss of stereocilia from longer row in OHC hair bundle. Scale bars: $2 \mu \mathrm{m}$ in C and right panels in $\mathbf{D}$, and $5 \mu \mathrm{m}$ in $\mathbf{D}$, left panel.

tural analyses were performed on separate TEM images (Figure 4, A-C) and on reconstructions of images of rootlets using both TEM sections (Figure 4, D-F, and Supplemental Figure 6) and consecutive backscattered SEM images after 20-nm-step milling with a focused ion beam (FIB-SEM, Figure 5 and Supplemental Videos 1 and 2). Compared with WT, by P16 hair cells from Triobp $p^{1 E x \cdot-10 / \triangle E x \cdot-10}$ mice showed a variety of structural defects in the upper half of a rootlet within the stereocilia F-actin core and also in the lower half of a rootlet that is embedded into the cuticular plate (Figure 4 and Supplemental Figure 6). Some stereocilia appeared to be missing the lower half of rootlets, while the other rootlets were thin, fragmented, and dysmorphic in their lower half within the cuticular plate. Sometimes rootlets had interruptions or breaks at sites where a stereocilium inserts into the cuticular plate (Figure 4E). Rootlet abnormalities in TRIOBP-5deficient mice occurred throughout the length of the cochlea, in all rows of stereocilia (Supplemental Figure 6). Earlier signs of rootlet abnormalities at P7-P9 (Supplemental Figure 7) included an expansion of the upper half of the rootlets within stereocilia cores and loss or thinning of the lower half of some rootlets within cuticular plates, similar to changes reported in cat and chinchilla after noise trauma (38-40).

FIB-SEM images confirmed these morphological findings and allowed for quantification of 3D structures of stereocilia rootlets (Figure 5 and Supplemental Videos 1 and 2). Rootlets of both IHCs and OHCs of P14 Triobp $p^{1 E x \cdot 10 / 1 \mathrm{E} x x^{-10}}$ mice were hypertrophic within stereocilia cores but abnormally thin, often bent, 
A

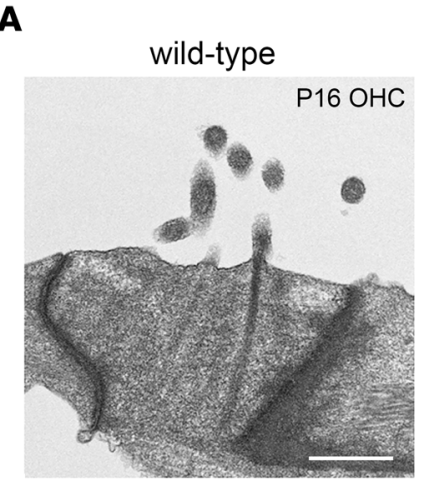

D

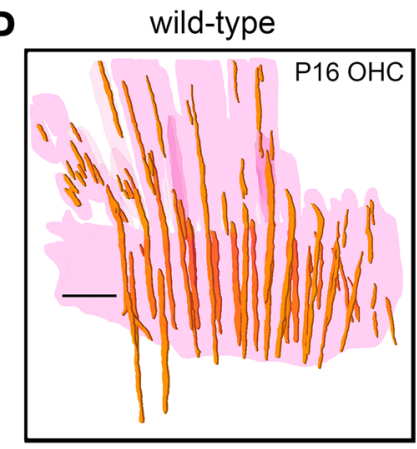

G

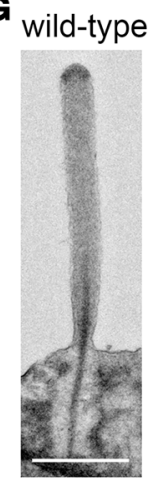

B

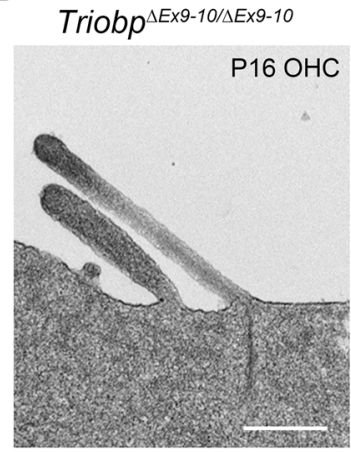

E Triobp ${ }^{\Delta E \times 9-10 / \Delta E \times 9-10}$

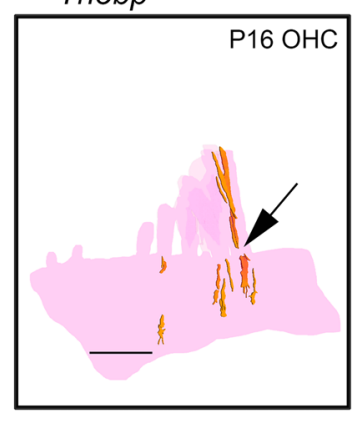

I
C

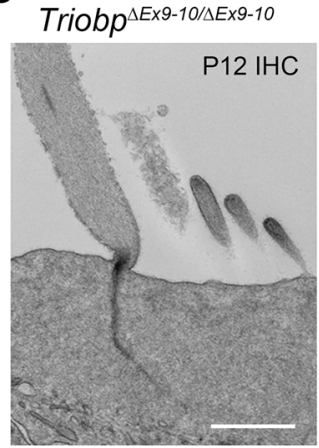

F TriObp ${ }^{\triangle E \times 9-10 / \triangle E \times 9-10}$

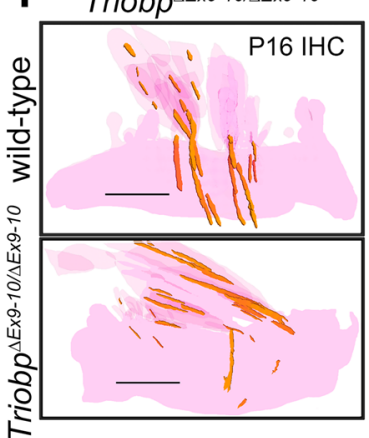

는
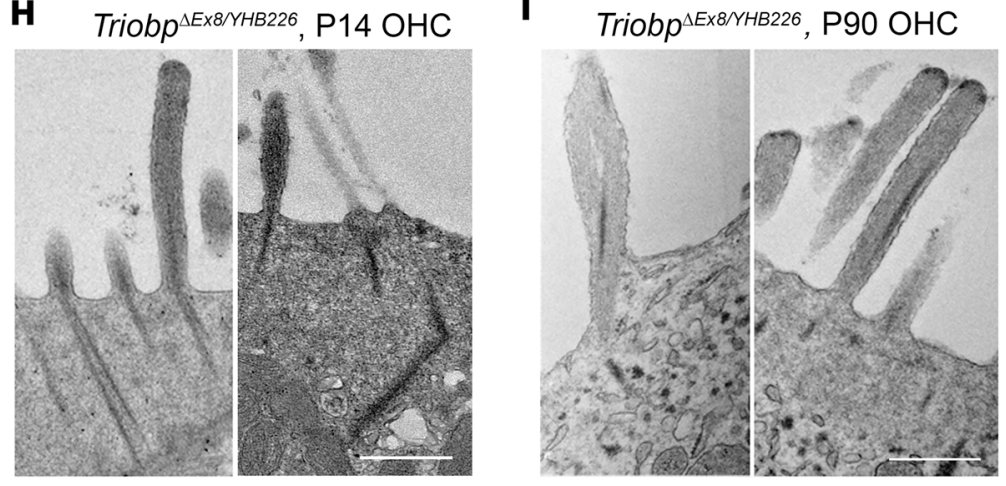

Figure 4. Structural abnormalities of stereocilia rootlets in TRIOBP-5-deficient mice. (A-C) TEM images of hair cell bundles (P12 and $\mathrm{P} 16)$ showing representative rootlet morphology and (D-F) 3D reconstructions of stereocilia rootlets from serial TEM sections (P16). wild-type $\left(\right.$ Triobp $\left.^{+/+}\right)$(A and $\left.\mathbf{D}\right)$ and Triobp ${ }^{4 \times \times 9-10 / 4 E \times 9-10} \mathrm{OHCs}$ (B and E), Triobp $p^{4 E \times 9-10 / 4 E \times 9-10} \mathrm{IHC}$ rootlet morphology (C), and 3D reconstruction of wild-type $\left(\right.$ Triobp $\left.^{+/+}\right)$and Triobp ${ }^{\Delta E \times 9-10 / 4 E \times 9-10} \mathrm{IHC}$ stereocilia and cuticular plates (F). (C) IHC stereocilium rootlet is dysmorphic, thin, and bent. (E) Arrow points to disrupted rootlet structure at stereocilia pivot points. (G-I) TEM images of the OHC stereocilia in wild-type (Triobp ${ }^{+/+}$) at P16 (C), and Triobp $p^{4 E \times 8 / \text { YHB226 }}$ mouse at P14 (H) and P90 (I). Structural defects of rootlets at P14 include splayed bundles of F-actin or bent rootlets (H). Representative structural defects at P9O include fused stereocilia undergoing degeneration (I, left panel) and abnormal asymmetric localization of electron dense material within stereocilia F-actin cores (I, right panel). Scale bars: $500 \mathrm{~nm}$.

and elongated within the cuticular plates (Figure 5, A-P). At P7-P14, rootlets of Triobp ${ }^{4 E x \cdot-10 / 1 E x 9-10} \mathrm{OHCs}$ within stereocilia cores were abnormally expanded, nearly reaching the tips of stereocilia (Figure 5, M-S, and Supplemental Figure 7). We concluded that TRIOBP-5 is required to establish the normal development and intricate architecture of rootlets within stereocilia cores and cuticular plates.

Compared with Triobp ${ }^{1 E x \cdot-10 / 1 E x \cdot 90}$, rootlets in Triobp ${ }^{1 E \times 8 / Y H B 226}$ mice were somewhat less affected. While some rootlets were bent, splayed, or had multiple misplaced fragments of rootlet-like dense structures within stereocilia cores, others were of normal thickness and length within the cuticular plate and in the stereocilia core (Figure 4, H and I). Additionally, Triobp ${ }^{4 E \times 8 / Y H B 226}$ rootlets degenerated more slowly than rootlets of Triobp ${ }^{1 E x \cdot-10 / 1 \mathrm{Ex} x \cdot 10}$ and persisted even at $\mathrm{P} 90$, although dysmorphic (Figure 4I). These observations suggest that the C-terminal portion of TRIOBP-5 contributes to the structural integrity of rootlets, perhaps by fortifying their F-actin bundles to withstand life-long acoustical stimulation. 
A

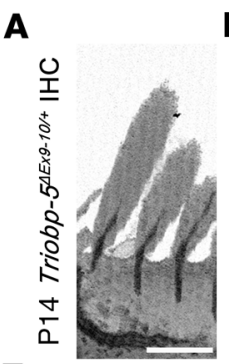

E

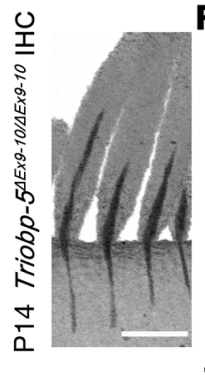

I

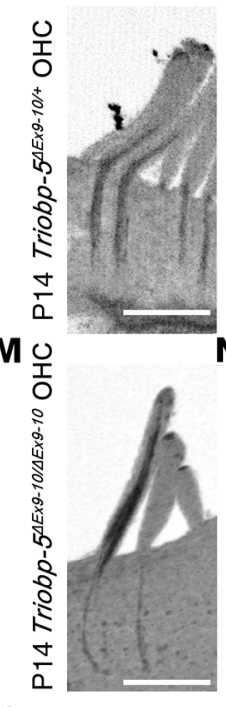

Q

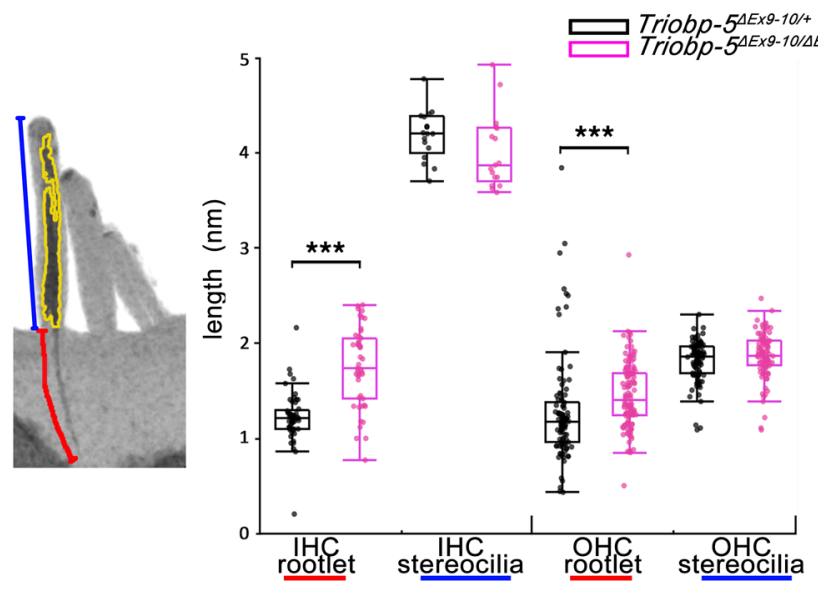

B

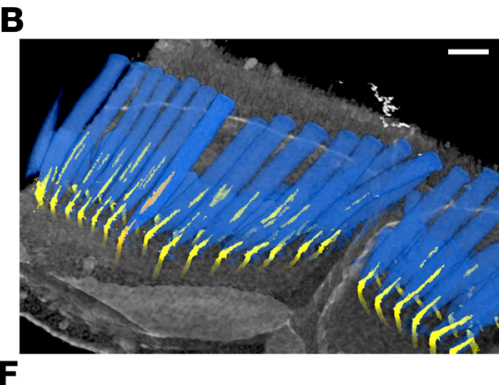

C
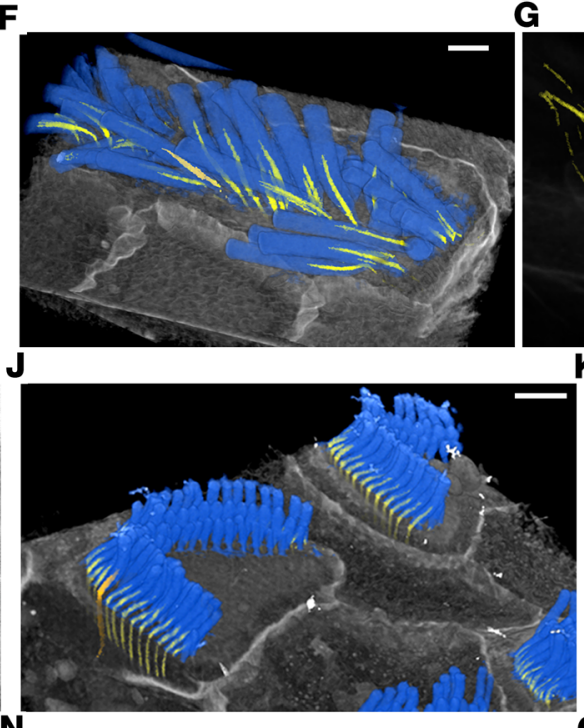
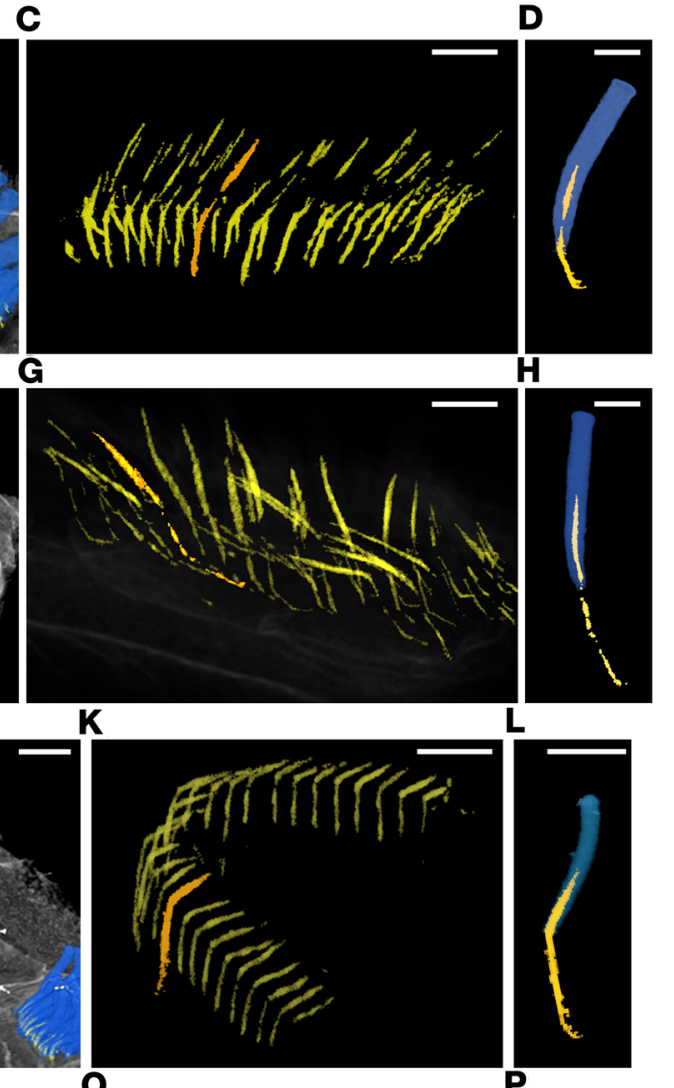

P

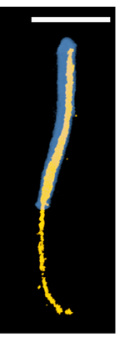

$\mathbf{S}$

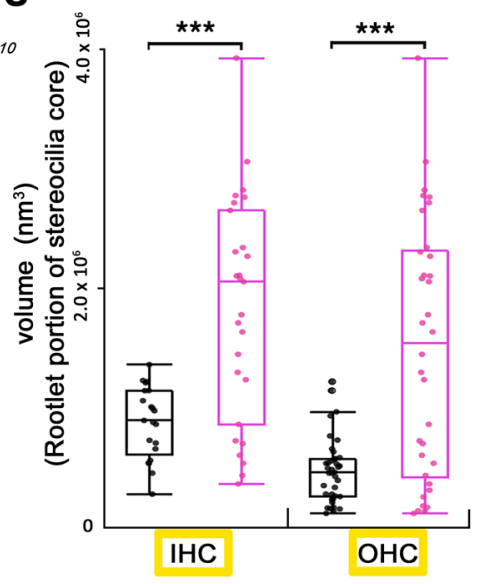


Figure 5. FIB-SEM analyses of Triobp ${ }^{4 x_{9}-10 / 4 E x 9-10}$ hair cells show enlargement of rootlets within stereocilia cores and thinning of rootlets within the cuticular plate. (A-P) Reconstruction of stereocilia bundles and stereocilia rootlets from P14 Triobp Exg-10/4Exg-10 and Triobp Exg-10/+ IHCs and OHCs. (A, E, I, and M) Sagittal sections showing the morphology of rootlets reconstructed from FIB-SEM data sets. (B, F, J, and $\mathbf{N}$ ) False-colored $3 D$ reconstructions of hair bundles (blue) and rootlets (yellow and orange) were added to better visualize rootlet structure. (C, G, K, and $\mathbf{O}$ ) Individual rootlets highlighted in orange show typical morphology for a particular genotype. (D, H, L, and $\mathbf{P}$ ) Reconstruction of 1 stereocilium (blue) and rootlet selected in $\mathbf{C}$, G, K, and $\mathbf{O}$ (orange) from hair cells in $\mathbf{B}, \mathbf{F}, \mathbf{J}$, and $\mathbf{N}$. The rootlet segments within stereocilia cores of Triobp Exg-10/4Exg-10 mice in $\mathbf{E - H}$ and $\mathbf{M}-\mathbf{P}$ are longer and thicker compared with phenotypically wild-type controls in A-D and I-L, while Triobp Ex9-10/4Exg-10 rootlets within the cuticular plate are abnormally thin. Scale bars: $1 \mu$ m. See also Supplemental Videos 1 and 2. (Q) Quantification of the shape of stereocilia and rootlets, rootlet length (red line), and stereocilium length (blue line) and volume of rootlet segment of stereocilia core (yellow) of the tallest row stereocilia of IHCs and OHCs. (R and S) Data in box-and-whisker plots are represented as mean \pm SD. Data points represent length of rootlets within the cuticular plate (red) and within the stereocilia cores (blue) of IHCs or OHCs and volume of rootlets within stereocilia cores for Triobp $-5^{4 E \times 9-10 /+}$ mice (black; $n=54,17,102,98,21$ and 39) or Triobp-5 27, and 34) at P14. ${ }^{* *} P<0.001$ compared with control by Mann-Whitney $U$ test. There is a significant difference in rootlet length and rootlet volume in stereocilia cores when comparing Triobp $-5^{\Delta E \times 9-10 /+}$ and Triobp- $5^{\Delta E \times 9-10 / 4 E \times 9-10}$ mice, while there is no significant difference in stereocilia length.

TRIOBP-4 is present in stereocilia of deaf TRIOBP-5-deficient mice. To examine the localization of TRIOBP-4 in the organ of Corti in the absence of WT TRIOBP-5, we performed immunostaining of Triobp ${ }^{4 E x 9-10 / 4 E x-10}$ and Triobp ${ }^{4 E \times 8 / Y H B 226}$ deaf mice. The anti-TRIOBP-5 antibody did not detect TRIOBP-5 in Triobp ${ }^{4 E x-10 / \triangle E x 9-10}$ hair cells (Figure 6, A-C, and Supplemental Figure 1), while the TRIOBP-4/5 antibody detected TRIOBP-4 in the cuticular plate and in stereocilia cores of both Triobp-5-deficient mouse models (Figure 6, D and E, and Supplemental Figure 5). However, the anti-TRIOBP-5 antiserum did stain stereocilia rootlets of compound heterozygous Triobp ${ }^{4 \text { Ex } 8 \text { YHB226 }}$ mice (Figure 6, F-H). RT-PCR analysis of Triobp ${ }^{4 E \times 8 / \text { YHB226 }}$ mouse inner ear mRNA detected Triobp-5 transcripts skipping exons 17 to 23 or 17 to 24 (Supplemental Figure 8). If translated, this transcript could explain the TRIOBP-5 immunoreactivity in the Triobp ${ }^{4 E x / / Y H B 226}$ stereocilia rootlets and the less severe deafness of the Triobp ${ }^{\triangle E x / / Y H B 226}$ as compared with the Triobp ${ }^{4 E x 9-10 / \triangle E x 9-10}$ mouse (Figure 3), but cannot substitute for the function of full-length TRIOBP-5 since a Triobp ${ }^{1 E \times 8 / Y H B 226}$ mouse eventually becomes profoundly deaf.

TRIOBP-5 homo-oligomerizes. Exons 18, 19, 21, 23, and 24 encode residues common to TRIOBP-1 and TRIOBP-5 that are predicted to form 5 coiled-coil domains (Figure 1A and Supplemental Figure 8). Some of these coiled-coils allow TRIOBP-1 to oligomerize (14). To gain additional insight into the domain architecture and possible dimerization of mouse TRIOBP-5, we performed an amino acid sequence analysis to identify putative coiled-coil domains using COILS (41). Five coiled-coil domains were identified at residues 1669-1689, 1719-1754, 1792-1819, 1890-1917, and 1928-1962 of mouse TRIOBP-5 (NCBI ABB59557.2), equivalent to the coiled-coil domains described for human TRIOBP-5 (14). Next, we generated an in silico template-based structural model (see Methods and Supplemental Figure 8) in order to analyze the 3D arrangement of these coiled-coil domains. Similar to that of the template, the resulting model has a dimer architecture containing 2 protein chains of the C-terminal domain of mouse TRIOBP-5 covering residues 1713-1868, which correspond to the predicted coiled-coils 2 and 3 (Figure 1A and Supplemental Figure 8).

To experimentally test predictions of the computational model that TRIOBP-5 can homo-oligomerize, we employed a NanoSPD assay, which utilizes a myosin-10 motor for a nanoscale pull down to detect a protein-protein interaction in live cells (42). The MYO10-HMM-Nanotrap (MYO10 NANOTRAP) construct was designed to bind specifically to GFP (it does not bind DsRed) and transport it to the tips of filopodia (42). HeLa cells were transfected simultaneously with 3 expression constructs: MYO10 ${ }^{\text {NANOTRAP }}$, AcGFP1-TRIOBP-5, and DsRed-TRIOBP-5. The MYO10 NANOTRAP bound AcGFP1-TRIOBP-5 and delivered it to filopodia tips. Yellow fluorescence at the tips indicated that DsRed-TRIOBP-5 interacted with AcGFP1-TRIOBP-5, and this macromolecular complex was shuttled to filopodia tips (Figure 7, A and C, and Supplemental Figure 9). In control experiments, we transfected the MYO10 NANOTRAP, DsRed-TRIOBP-5, and AcGFP1 expression vectors and observed only GFP fluorescence at the tips of filopodia, indicating that neither MYO10 ${ }^{\text {NANOTRAP }}$ nor the AcGFP1 vector were able to interact with DsRed-TRIOBP-5 and traffic it to filopodia tips (Figure 7, A and C). As an additional control, AcGFP1-TRIOBP-5 coexpressed with DsRed-TRIOBP-5, but without MYO10 NANO TRAP, colocalized within the cytoplasm, forming punctate structures that were not observed at filopodia tips (Supplemental Figure 9). DsRed-TRIOBP-5 alone was only present in the cytoplasm as punctate structures (Supplemental Figure 9), arguing for the necessity of the active transport of this protein to filopodia tips by the myosin-10 motor in conjunction with bound AcGFP1-TRIOBP-5. To determine if homo-oligomerization of TRIOBP-5 depends on the coiled-coil domains, the sequence encoding the coiled-coil domains was deleted from TRIOBP-5. This internally deleted TRIOBP-5 variant failed to homo-oligomerize (Figure 7, B and D), consistent with the computational structural model showing that the coiled-coil domains permit homo-oligomerization (Figure 7 and Supplemental Figure 8). 


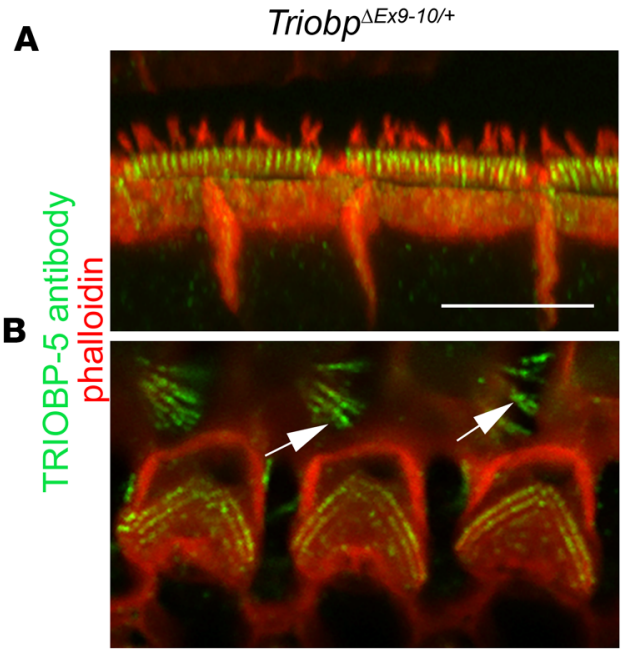

c

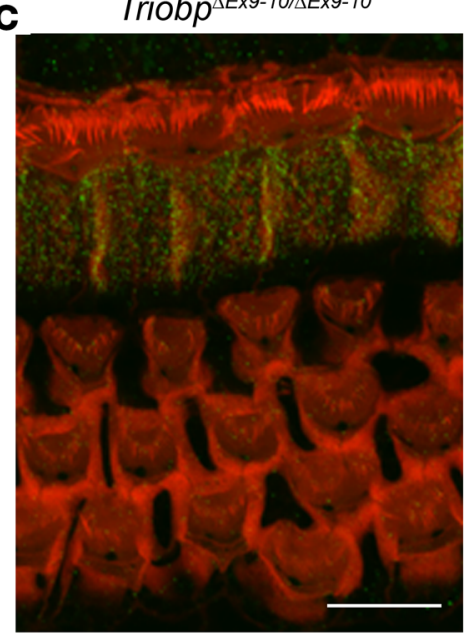

D

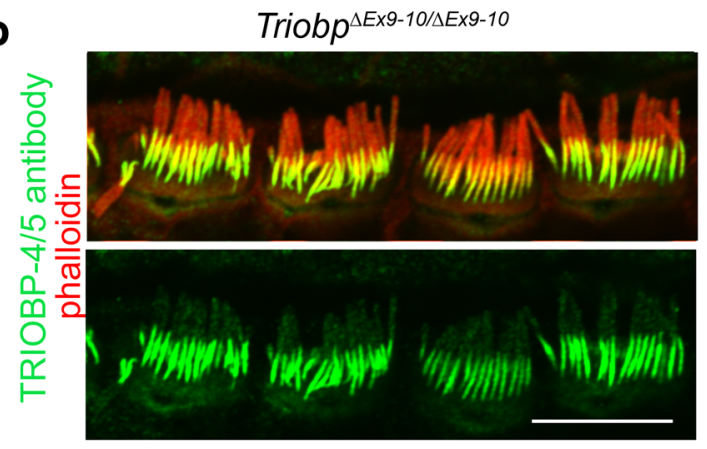

$\mathbf{F}$

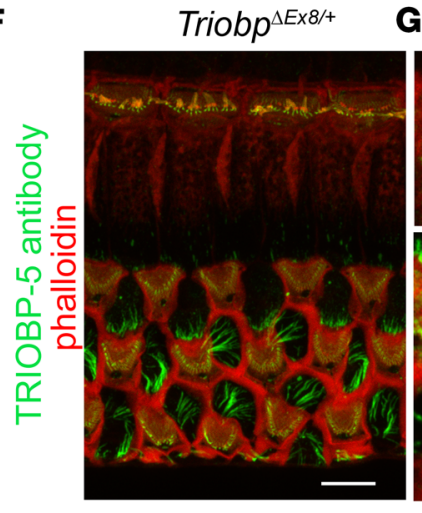

E Triobp $p^{\triangle E \times 9-10 / \Delta E \times 9-10}$

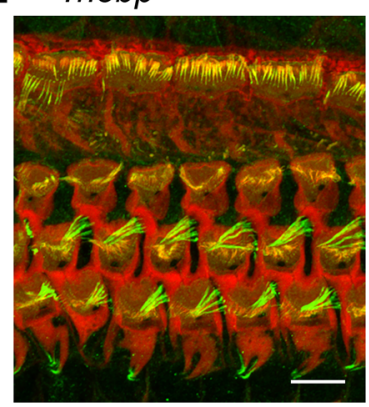

H Triobp ${ }^{\triangle E \times 8 / Y H B 226}$

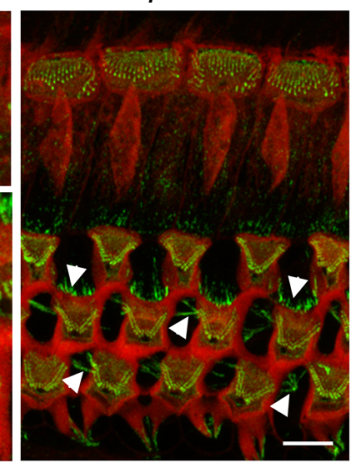

Figure 6. Localization of TRIOBP isoforms in P14P30 TRIOBP-5-deficient mice. (A and B) TRIOBP-5 (green) is localized to the rootlet compartment within the cuticular plate of IHCs (A) and $\mathrm{OHCs}$ and in Deiters' supporting cells (B, white arrows) of normal-hearing Triobp ${ }^{4 \mathrm{Ex}-10 /+}$ mice. (C) TRIOBP-5 was not detected in stereocilia rootlets and Deiters' cells of Triobp $p^{\Delta E x 9-10 / \Delta E \times 9-10}$ homozygous TRIOBP-5deficient mice, confirming antibody specificity. (D) TRIOBP-4/5 antibody detects the TRIOBP-4 isoform in Triobp ${ }^{4 \times \times 9-10 / 4 E \times 9-10} \mathrm{IHC}$ stereocilia rootlet compartment above the cuticular plate. (E) Immunoreactivity to TRIOBP-4/5 antibody is unchanged in Deiters' cells and OHCs of Triobp $p^{4 \times 99-10 / 4 E \times 9-10}$ mice compared to immunoreactivity in phenotypically wild-type heterozygous (Triobp ${ }^{\Delta E \times 9-10 /+}$ ) hair cells (Figure 2B). (F-H) In contrast to the absence of a TRIOBP-5 signal in Triobp ${ }^{4 \mathrm{Exg}-10 / \Delta E \times 9-10}$ mice, TRIOBP-5 antibody shows immunoreactivity in compound heterozygous Triobp ${ }^{4 E \times 8 / Y H B 226}$ and control Triobp ${ }^{4 \times \times 8 /+}$ mice in the lower portion of stereocilia rootlets in both OHCs and IHCs (arrows in $\mathbf{G}$ ) and in Deiters' and outer pillar cells (arrowheads in $\mathbf{G}$ and H). Scale bars: $5 \mu \mathrm{m}$ (all panels).

TRIOBP-5 deficiency causes decreased apical stiffness of supporting cells. TRIOBP-4 and TRIOBP-5 are also expressed in sensory epithelium supporting cells and are localized to filamentous structures within the obliquely ascending Deiters' processes and apical plates of outer pillar cells (Figure 1B; Figure 2, A and B; and Figure 6B). In Tri$o b p^{\Delta E x 9-10 / \Delta E x 9-10}$ mice, the TRIOBP-5 antibody signal is absent from supporting cells (Figure 6C). We tested the hypothesis that TRIOBP contributes to the stiffness of the sensory epithelium by investigating the mechanical properties of the reticular lamina using PeakForce Tapping-mode atomic force microscopy (PFT-AFM), which allows simultaneous visualization and measurements of stiffness by gently indenting from $50 \mathrm{~nm}$ to no more than $500 \mathrm{~nm}$ the surface of live organ of Corti explants. The elastic Young's modulus $(E)(43)$ of the epithelium was measured, and nanoscale stiffness maps of P5 organ of Corti (middle turn) of mutant Triobp ${ }^{4 E x-10 / \Delta E x-10}$ and normal-hearing heterozygous Triobp $p^{4 \mathrm{Ex}-10 /+}$ littermates were generated (Figure 8A). At apical surfaces of pillar and Deiters cells of Triobp ${ }^{4 E x 9-10 / \triangle E x 9-10}$ mice, local stiffness of the reticular lamina was reduced approximately 2-fold compared with heterozygotes (Figure 8B and Supplemental Table 1). Thus, alteration of these filamentous structures in supporting cells deficient for TRIOBP-5 (Figure 9A) reduced axial compliance of the organ of Corti reticular lamina, likely affecting hearing sensitivity $(21,44,45)$.

Pivotal flexibility of stereocilia is diminished in TRIOBP-5-deficient hair cells. The contribution of TRIOBP-5 to the pivotal flexibility and durability of stereocilia was investigated by deflecting stereocilia bundles of live IHCs using stimuli of increasing intensity from a calibrated fluid-jet (Figure 8C). In WT IHCs, hair bundle deflections depended almost linearly on the pressure of the fluid-jet (Figure 8D). Deflections of the Triobp ${ }^{4 E x 9-10 / \triangle E x 9-10} \mathrm{IHC}$ bundles did not differ from WT at low stimuli intensities. However, at higher stimuli intensities, the extent of the deflection of Triobp ${ }^{4 E x 9-10 / 4 E x 9-10}$ IHC bundles was significantly larger compared with WT (Figure 8D and Supplemental Video 3). This nonlinear 
A
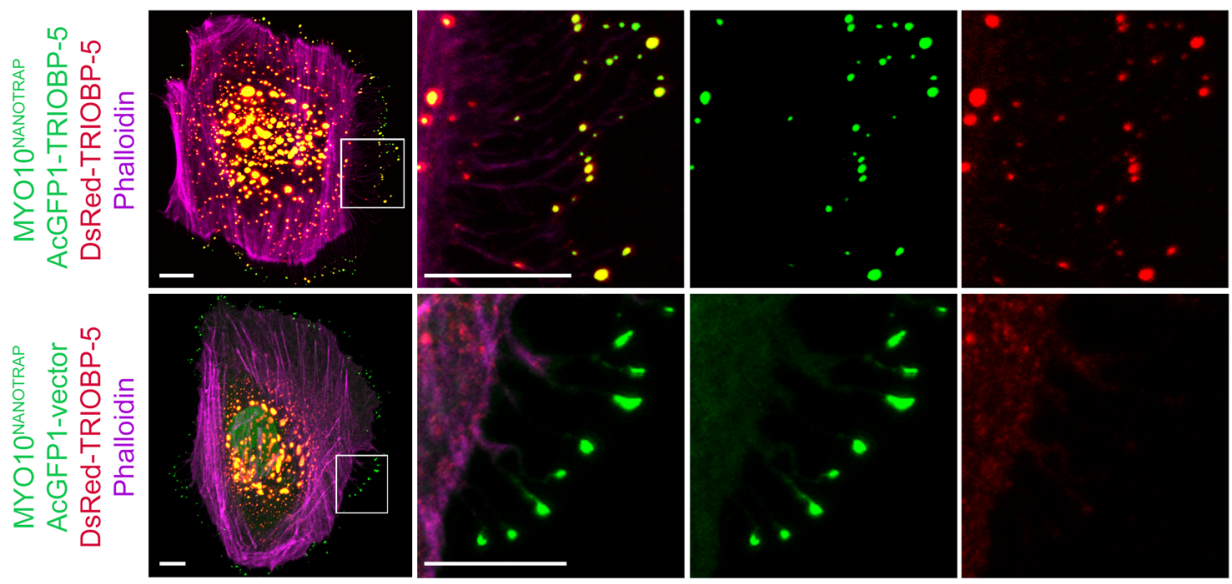

B
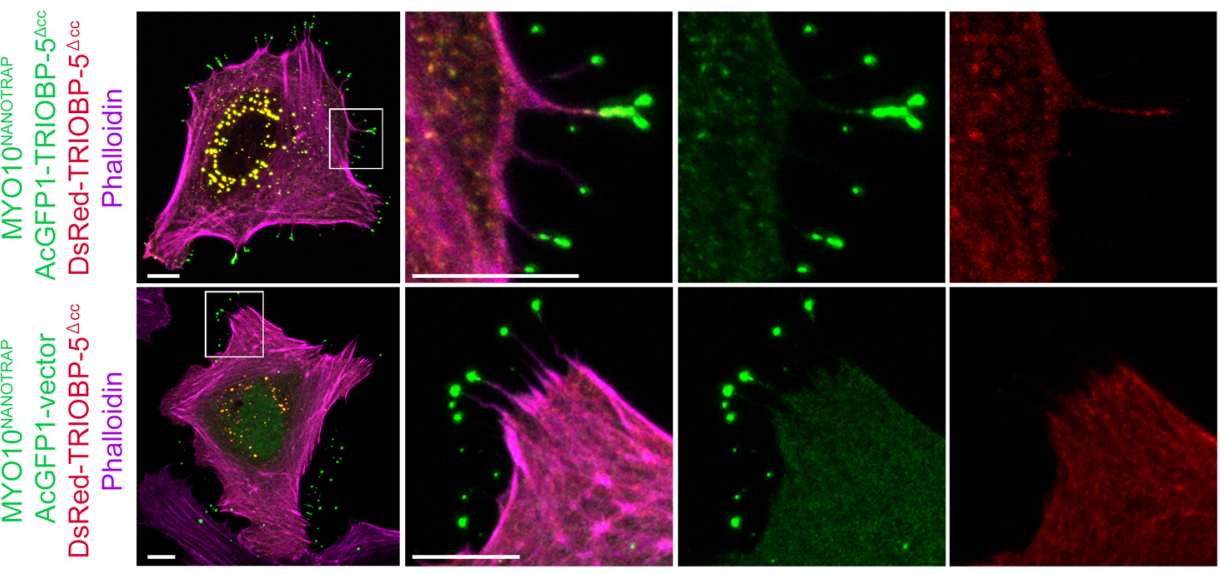

C

MYO10 NANOTRAP + DsRed-TRIOBP-5 prey

D
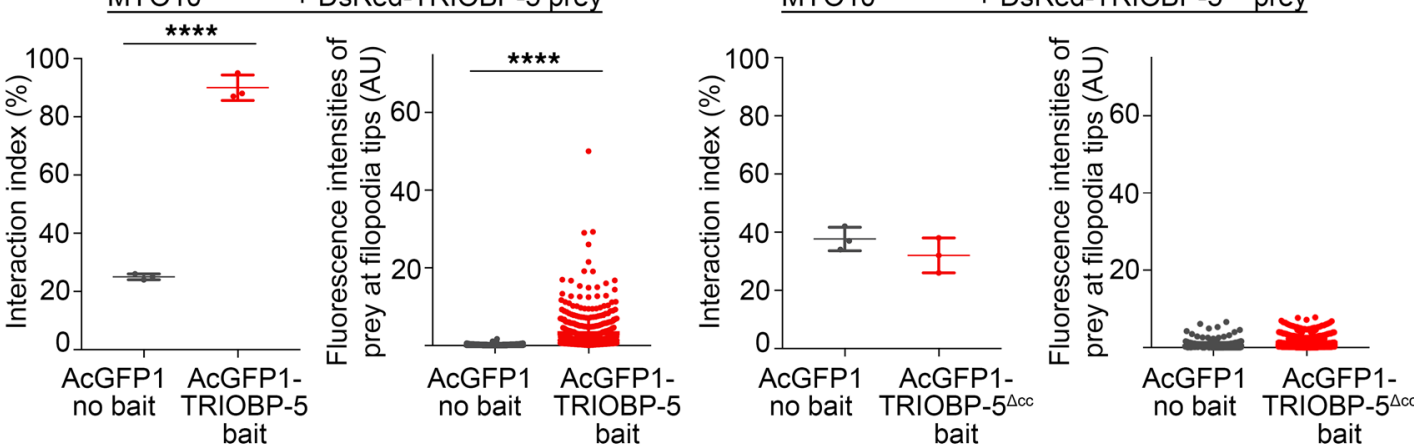

Figure 7. TRIOBP-5 homodimerization revealed by nanoscale pull-down assays. (A) MYO10-HMM-Nanotrap (abbreviated MYO10 NANOTRAP) Specifically binds GFP derivatives, but not DsRed, and traffics GFP-tagged protein to filopodia tips. Representative image of a HeLa cell transfected with 3 expression vectors encoding MY010 ${ }^{\text {NANOTRAP }}$, AcGFP1-TRIOBP-5, and DsRed-TRIOBP-5 (upper panel), and a control HeLa cell transfected with the MYO10 ${ }^{\text {NANOTRAP }}$ AcGFP1 vector, and DsRed-TRIOBP-5 (lower panel). AcGFP1-TRIOBP-5 and DsRed-TRIOBP-5 colocalize to filopodia tips (upper panel), while in the control, only the AcGFP1 vector localized at filopodia tips (lower panel). The complex of MY010 NANOTRAP and AcGFP1-TRIOBP-5 partnered with and transported DsRed-TRIOBP-5 to filopodia tips. The complex of MYO10 ${ }^{\text {NANOTRAP }}$ and AcGFP1 vector did not traffic DsRed-TRIOBP-5. These data indicate that TRIOBP-5 can homomultimerize in vivo. The second column shows enlarged images of the white-outlined areas from the first column. The third and fourth columns are the individual green and red channels of merged images in the second column. (B) Transfected HeLa cell with MYO10 NANOTRAP, AcGFP1-TRIOBP-5 ${ }^{\triangle c c}$, and DsRed-TRIOBP-5 $5^{\Delta c c}$ (upper panel), and control HeLa cell transfected with MY010 ${ }^{\text {NANOTRAP }}$, AcGFP1 vector, and DsRed-TRIOBP-5 ${ }^{\Delta c c}$ (lower panel). Triobp-5 $5^{\Delta c c}$ is deleted for sequence encoding the coiled-coil domains and $78 \mathrm{bp}$ of upstream sequence. DsRed-TRIOBP-5 $5^{\Delta c c}$ was not trafficked to filopodia tips by the MYO10 ${ }^{\text {NANOTRAP }}$ and AcGFP1-TRIOBP-5 ${ }^{\Delta c c}$, indicating that TRIOBP-5 ${ }^{\triangle c c}$ does not multimerize. (C) Left panel: Quantification of AcGFP1-TRIOBP-5 or AcGFP1 vector binding to DsRed-TRIOBP-5. When MY010 NANOTRAP, AcGFP1-TRIOBP-5, and DsRed-TRIOBP-5 were coexpressed, $90 \% \pm 4.4 \%$ of filopodia (3 independent experiments) showed a statistically significant correlation between green and red fluorescence at filopodia tips (2-tailed $t$ test). Right panel: Fluorescence intensities of DsRed-TRIOBP-5 at filopodia tips. The presence of AcGFP1-TRIOBP-5 caused a significant increase of fluorescence intensity of DsRed-TRIOBP-5 at the filopodia tips (Mann-Whitney $U$ test). (D) Interaction index of either AcGFP1-TRIOBP-5 $5^{\Delta c c}$ or AcGFP1 vector with DsRed-TRIOBP-5 ${ }^{\Delta c c}$ (left panel). AcGFP1-TRIOBP-5 ${ }^{\Delta c c}$ does not bind to DsRed-TRIOBP-5 ${ }^{\Delta c c}$. Fluorescence intensities of DsRed-TRIOBP-5 ${ }^{\Delta c c}$ at filopodia tips (right panel). AcGFP1-TRIOBP-5 $5^{\Delta c c}$ does not increase the intensity of DsRed-TRIOBP-5 $5^{\Delta c c}$ at filopodia tips, indistinguishable from the AcGFP1 vector control experiment. Scale bars: $10 \mu \mathrm{m}$. Data are mean \pm SD. ${ }^{* * *} P<0.0001$. 
A
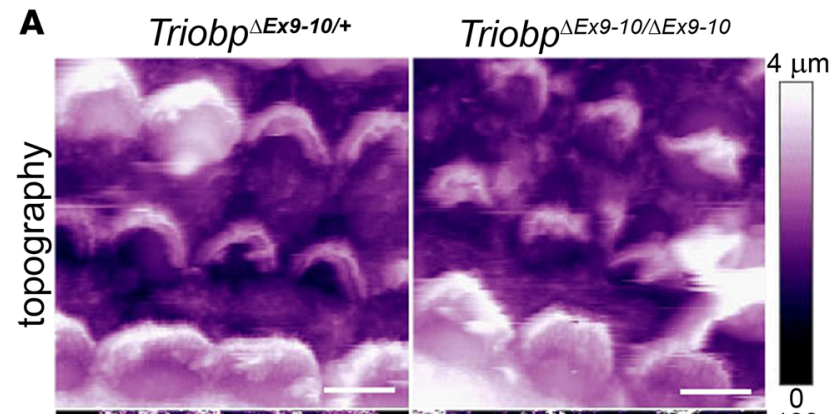

Triobp
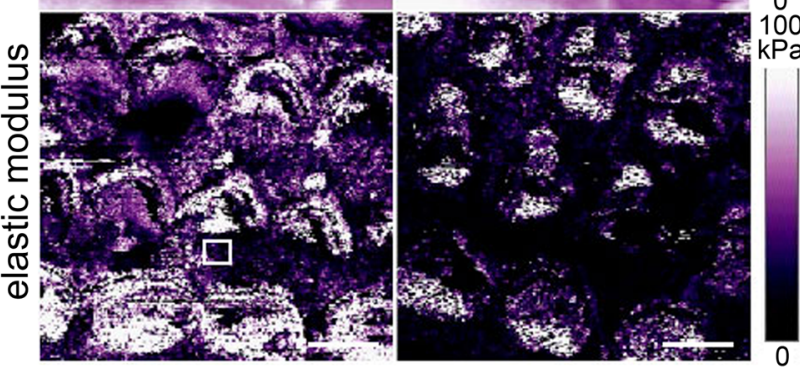

C
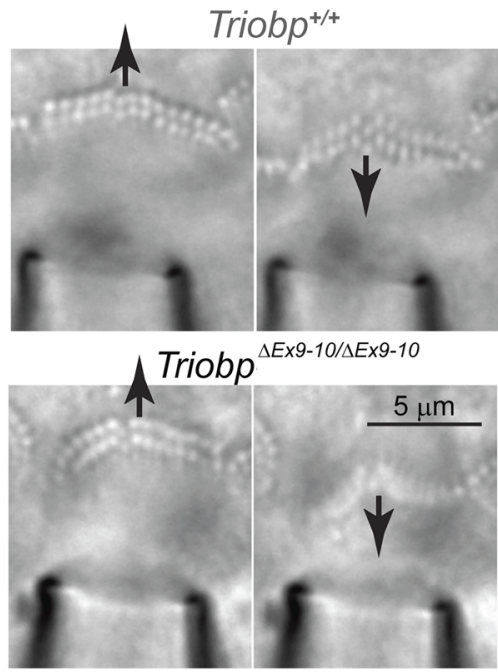

D
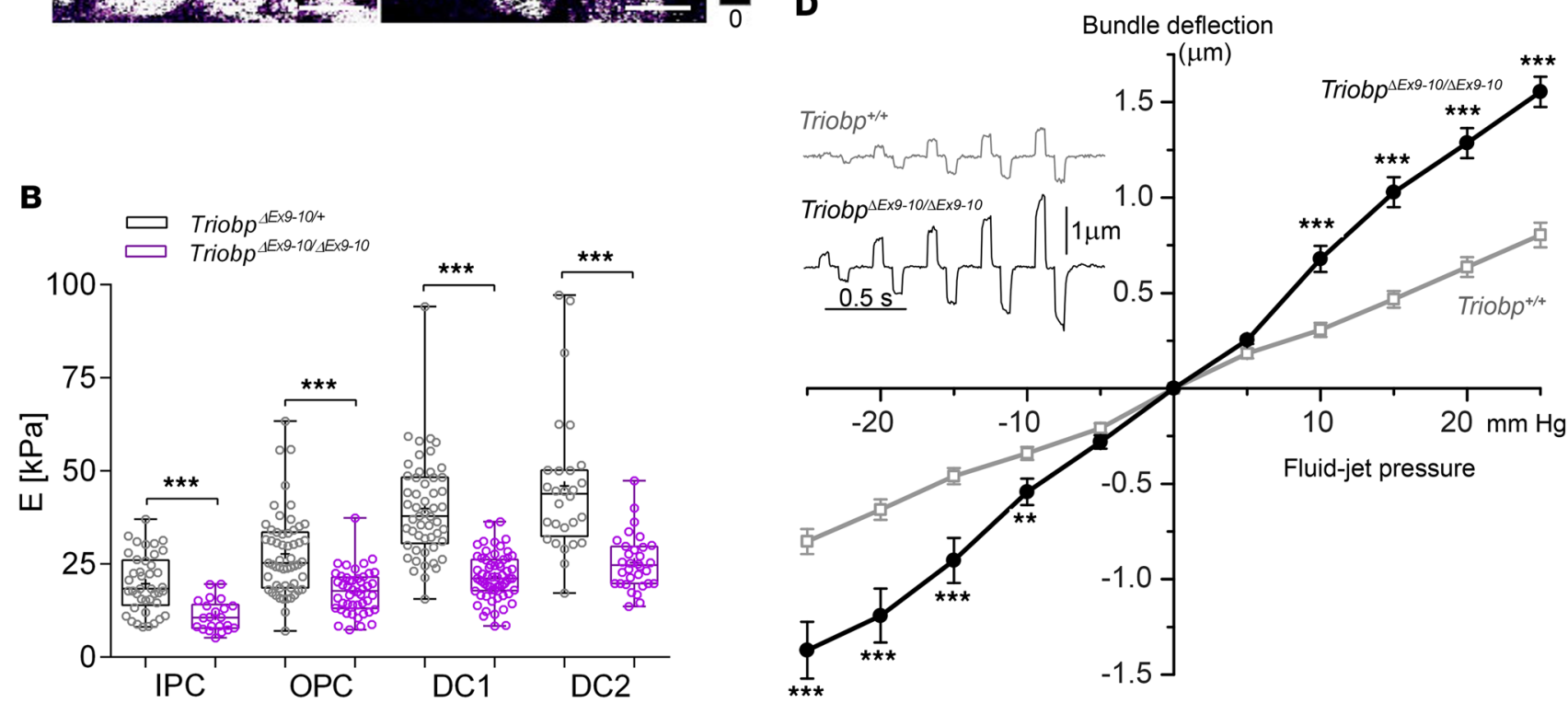

Figure 8. TRIOBP-5 deficiency results in decreased supporting-cell stiffness and fragile hair cell stereocilia. (A) Topographic image (top) and stiffness map (bottom) of live organ of Corti explants (P5, cochlear middle turn) from Triobp ${ }^{4 \mathrm{E} \times 9-10 /+}$ heterozygotes (left) and Triobp ${ }^{4 E \times 9-10 / \triangle E \times 9-10}$ mutant littermates (right) visualized by atomic force microscopy using PeakForce Tapping mode (PFT-AFM). Transverse stiffness (E, elastic Young's modulus) of the reticular lamina measured in regions of interest (ROIs) overlaying supporting cells (for example, white square in Triobp ${ }^{\Delta E \times 9-10 / 4 E \times 9-10}$ stiffness image). Scale bars: $5 \mu$ m. (B) Box-and-whisker plots of $E$ values on apical surfaces of inner pillar (IPC), outer pillar (OPC), and Deiters' row 1 (DC1) and 2 (DC2) cells. Bars show mean \pm SD Asterisks indicate statistical significance between Triobp- $5^{+/ 4 E x g-10}$ and Triobp-5 $5^{\Delta E \times 9-10 / 4 E \times 9-10}$ cells. ${ }^{* * *} P<0.001$ (2-tailed $t$ test with Welch's correction). $n$ indicates the number of cells. Data points represent individual supporting cells for Triobp ${ }^{4 E \times 9-10 /+}$ mice (gray) and Triobp ${ }^{4 \times \times 9-10 / 4 E \times 9-10}$ mice (purple), 5 and 4 mice, respectively. (C) Deflections of stereocilia bundles by a fluid-jet in wild-type (top) and Triobp ${ }^{4 \times x 9-10 / 4 E \times 9-10}$ live IHCs (bottom). Responses to maximal pressure steps of $\pm 25 \mathrm{mmHg}$ are shown. See also Supplemental Video 3. The puff pipette is visible at the bottom of the panels. (D) Average displacements of stereocilia bundles as a function of fluid-jet pressure in wild-type (gray open symbols) and Triobp ${ }^{4 E \times 9-10 / 4 E x-10}$ (black solid symbols) IHCs at P8. Alternating positive and negative stimuli presented in increasing order. Examples of bundle deflections are shown in inset. Number of cells (number of mice): wildtype, $n=44$ (6); Triobp ${ }^{4 \times 99-10 / 4 E \times 9-10} n=49$ (6). Data are shown as mean \pm SEM. Asterisks indicate statistical significance of differences between wild-type and Triobp ${ }^{4 \times \times 9-10 / 4 E \times 9-10}$ mice. ${ }^{* *} P<0.01 ;{ }^{* *} P<0.001$ ( $t$ test of independent variables).

pressure-deflection relationship of Triobp ${ }^{4 E x 9-10 / A E x \cdot-10}$ stereocilia recapitulates the mechanical behavior of the IHC bundles in mice lacking both TRIOBP-4 and TRIOBP-5 (8). Interestingly, evidence for more fragile stereocilia of Triobp ${ }^{4 E x \cdot-10 / 1 E x \times-10}$ mice could be found not only in direct fluid-jet experiments (Figure 8D) but also in the PFT-AFM stiffness map data (Figure 8A). When a PFT-AFM probe lands on the tips of WT stereocilia, it generates peaks in the stiffness map that "outline" the hair bundle (Figure 8A). Although it is hard to interpret the values of these peaks due to a complex mechanical 
A
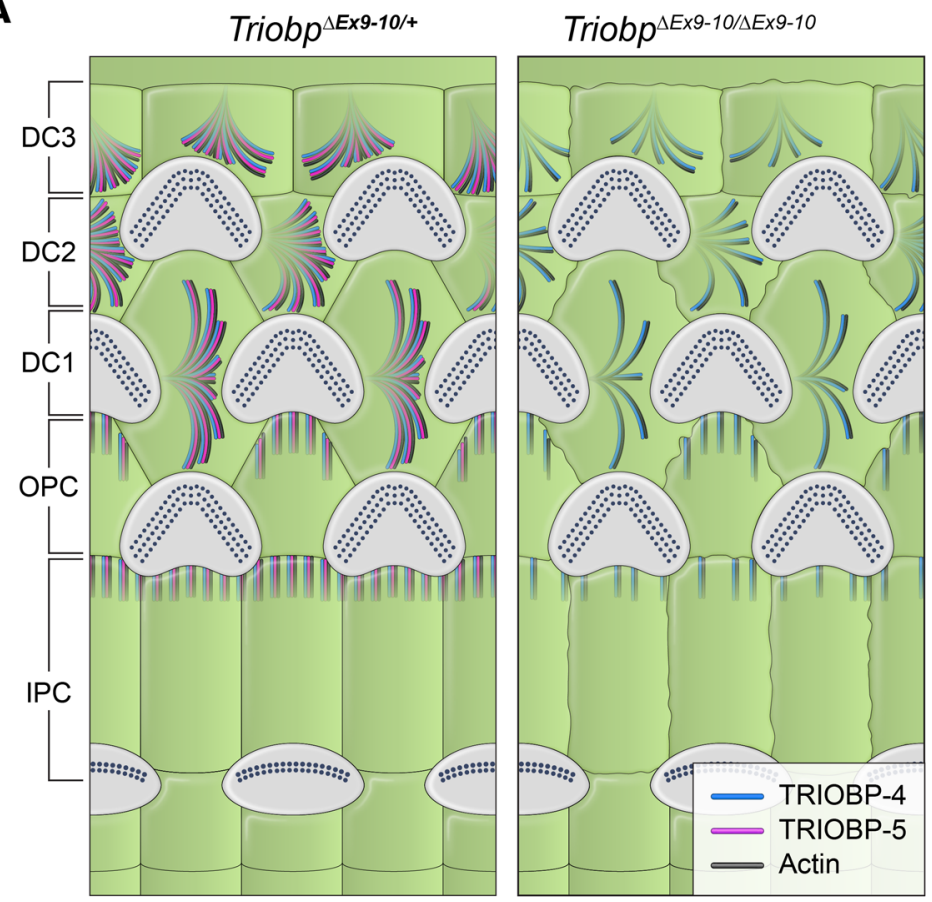

B

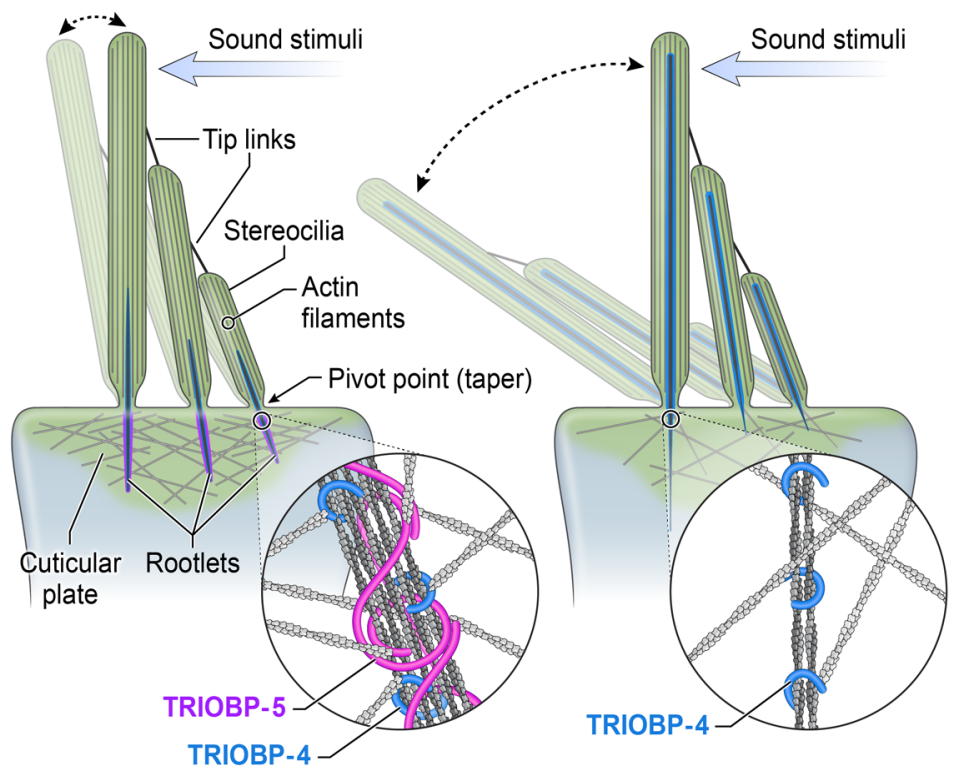

Figure 9. Illustration of TRIOBP-4 and TRIOBP-5 distribution in inner ear supporting cells and working model of stereocilia pathology in TRIOBP-5-deficient mice. (A) TRIOBP-4 and TRIOBP-5 are both present in Deiters' (DC1, DC2, and $D C 3)$ and outer and inner pillar cells (OPC, IPC) of normal-hearing Triob$p^{\text {SEx9-10/+ }}$ heterozygotes. Only TRIOBP-4 remains in the same cell types of deaf Triobp ${ }^{\triangle E \times 9-10 / \triangle E \times 9-10}$ mouse.

(B) Schematic of wild-type stereocilia with normal rootlet architecture (left). TRIOBP-5-deficient stereocilia with abnormally thin rootlets in the cuticular plate (right) but abnormally thick rootlets in the F-actin core that extend aberrantly to tips of stereocilia (right). TRIOBP-5 (purple) and TRIOBP-4 (blue) wrap around rootlet F-actin (insets) and in wild-type are hypothesized to allow filaments to slide past one another during sound-induced stereocilia deflections. TRIOBP-5 recruits more F-actin to shape rootlets and generates thicker bundles due to oligomerization by its coiled-coil domains.

interaction between the PFT-AMF probe and a stereocilium, they largely disappear in Triobp $p^{4 E x 9-10 / \Delta E x 9-10}$ mice (Figure $8 \mathrm{~A}$, bottom right), also indicating that a TRIOBP-5 deficiency affects the mechanical properties of stereocilia. Thus, 2 independent techniques revealed similar deficits in the mechanical properties of stereocilia bundles.

\section{Discussion}

The inner ear sensory epithelium must endure unremitting, life-long sound vibrations. Stereocilia rootlets of hair cells are one of the essential structures that provide this resilience. The absence of stereocilia rootlets causes deafness due to the degeneration of mechanosensory hair cells. We show here that the development of fully functional, morphologically distinctive stereocilia rootlets requires the specific functions of TRIOBP-5. Although essential, TRIOBP-4 is not sufficient to develop and sustain mature rootlets. Using 2 newly generated deaf mouse models, we demonstrated that TRIOBP-4 facilitates the initial formation 
of rootlets by promoting elongation into thin, tight bundles of F-actin, while TRIOBP-5 is essential for widening, sculpting, and then maintaining the intricate rootlet architecture. This study also provides the first evidence to our knowledge for a molecular subdivision of stereocilia rootlets of mammalian hair cells despite their uniform appearance in electron micrographs (26). The upper half of the rootlet within the stereocilium consists of actin filaments bundled predominantly by TRIOBP-4, while F-actin in the lower half of rootlets within the cuticular plate is bundled by both TRIOBP-4 and TRIOBP-5 (Figure 9B).

Elimination of TRIOBP-5 results in hypertrophy of the upper half of the rootlet and a thin, fragile lower half, while loss of just the C-terminal coiled-coil domains of TRIOBP-5 leads to easily damaged, bent, or splayed rootlets that have normal lengths. The TRIOBP-5 variant lacking coiled-coils, which is presumably responsible for the fluorescent signal in rootlets of Triobp ${ }^{4 E \times 8 / Y H B 226}$ mice, cannot fully compensate for the function of fulllength TRIOBP-5. This shorter version of TRIOBP-5 includes a PH domain and flanking sequence that fosters the mature shape of rootlets, restrains expansion into the upper half of stereocilia, and recruits additional actin filaments to thicken the lower half, but is not sufficient to provide resilience to stereocilia rootlets.

Using live cell NanoSPD assays, we show that coiled-coil domains are essential for oligomerization of TRIOBP-5, which appears to be a mechanism for holding actin bundles together. We propose that oligomerized TRIOBP-5 embraces filaments, allowing them to slide past one another within the bundle, thus providing flexible durability of rootlets during life-long deflection of stereocilia $(8,18)$. We also speculate that oligomerized TRIOBP-5 interacts with and integrates rootlets into the actin meshwork of the cuticular plate (Figure 9B), a function not fulfilled by TRIOBP-4. However, TRIOBP-4 is diffusely distributed within the cuticular plate and could help to organize its actin meshwork (Figure 9B). Because almost the entire sequence of TRIOBP-1 is identical to the sequence of the C-terminal part of TRIOBP-5, we could not exclude hetero-oligomerization of TRIOBP-5 and TRIOBP-1. However, in mice deficient for TRIOBP-4 andTRIOBP-5, butexpressing TRIOBP-1, rootlets donotdevelop(8). A conditionalknockout of TRIOBP-1 limited to hair cells is necessary to determine if TRIOBP-1 has a role in rootlet development or maintenance. Open questions also include the mechanisms targeting TRIOBP-4 and TRIOBP-5 to different compartments within stereocilia rootlets. Nevertheless, our observations indicate that the functions of TRIOBP-5 in rootlets are distinct from those of TRIOBP-4 and likely depend on its C-terminal actin-binding sites, $\mathrm{PH}$ domain, coiled-coil domains, or a combination of these functional modules (Figure 1A).

TRIOBP-5 is also essential for stiffening the reticular lamina, the tightly connected apical surfaces of sensory and supporting cells in the organ of Corti (34). Expression of TRIOBP-5 in the apical poles of the ascending Deiters' cell processes and in outer pillar cells near contacts with the cuticular plates of OHCs (Figure 1B and Figure 9A) contributes to mechanical stiffness and resilience of the auditory sensory epithelium. Using PFT-AFM, we observed that the absence of TRIOBP-5 in the organ of Corti resulted in a significant decrease in the local axial stiffness of the supporting cells within the reticular lamina. Thus, in addition to hair cell stereocilia rootlets, TRIOBP-5 provides mechanical resilience to Deiters' and pillar supporting cells of the organ of Corti that are also crucial for normal sound transduction.

In humans, a premature translation termination mutation (p.Gly1672*) in the sequence unique to TRIOBP-5 or 2 polymorphic variants of TRIOBP associate with moderate, progressive hearing loss (7), a phenotype similar to the progressive loss of hearing in our TRIOBP-5-deficient mouse and human age-related hearing loss, a common disorder in older adults (3). Over a lifetime, amino acid substitutions of TRIOBP protein or regulatory changes of the TRIOBP gene might subtly alter rootlet structure or rigidity of the apical surface of supporting cells, gradually compromising the armamentaria that safeguard hair cells against damage from sound-induced mechanical forces. In summary, our integrated genetic, morphological, physiological, and biophysical studies have revealed what we believe are unique F-actin cytoskeletal localizations and mechanisms of action of TRIOBP-5 and TRIOBP-4 within the inner ear sensory and non-sensory cells that in concert are required for normal hearing. The mechanisms of F-actin bundle formation by TRIOBP-5 and TRIOBP-4 isoforms uncovered in our study of deafness may help reveal the pathophysiology of other TRIOBP-associated disorders (TRIOBPopathies) and facilitate development of therapies.

\section{Methods}

R26-EGFP-Triobp-4 reporter mice. The R26-EGFP-Triobp-4 reporter mouse (accession no. CDB0289K: http:// www2.clst.riken.jp/arg/reporter_mice.html) was generated from $R 26-E G F P$-Triobp-4 conditional reporter 
mice (loxP-flanked STOP sequence in front of EGFP-Triobp-4 cDNA, accession no. CDB0280K) and backcrossed for 8 generations. In this study, our EGFP-Triobp-4 reporter is expressed under the ubiquitous transcriptional machinery of the noncoding RNA of the ROSA26 locus (46).

TRIOBP-5-deficient (Triobp ${ }^{4 E x-10 / \triangle E x 9-10}$ ) mice. The hearing phenotype of a mouse deficient only for the TRIOBP-5 isoform is unknown. Therefore, we used 2 different genetic strategies to engineer 2 new mutant mouse models, each deficient only for TRIOBP-5 (Figure 1A), which were backcrossed for more than 10 generations. The first 8 exons of mouse Triobp comprise the entire Triobp- 4 transcript and also contribute to the $5^{\prime}$ end of the much longer Triobp-5 transcript. The sequence of exons 13 through 25 are common to both Triobp-5 and Triobp-1 transcripts (Figure 1A), while exons 9 to 12 are exclusive to Triobp-5 transcripts (NM_001039156).

One TRIOBP-5-deficient mouse model was engineered by deleting exons 9 and 10 (Triobp $p^{4 E x-10}$ ), using a reported methodology (47). An 8-kb 5' arm and a 4-kb 3' arm for construction of the targeting vector were obtained from clone RP23-414K1 (BACPAC Resources Center). Except for the first 7 bp of Triobp exon 9 $(112 \mathrm{bp})$ and 1 base at the $3^{\prime}$ end of exon 10 (1021 bp), the remainder of the deleted sequences of these 2 exons was replaced with an $\mathrm{nLacZ}$ reporter cassette inserted in frame with the upstream protein sequence of TRIOBP. TT2 ES cells (48) were electroporated with the NotI-linearized targeting vector, and ES clones were screened for homologous recombination events. PCR-positive ES cell clones were then evaluated by genomic Southern blot analyses using specific probes (5' probe: 5'-CTACCCAGTCAGGCTTTGTTTTGTGGC-3' and 5'-GCCAATGTGAACTGCTTAACTC-3'; 3' probe: 5'-CTTGGTTTTTAATGTTAGAACCAGATTGGC-3' and 5'-CTAACCATCAGCTCATTTCTTTGAG-3'). Three independent ES cell clones (numbers 18, 20, and 23) were selected and used to produce chimeric mice. Germ-line transmission of the targeted allele was obtained from all 3 lines. Mice engineered with the deletion of exon 9 and 10 are designated Triobp ${ }^{4 E x 9-10}$, while the JAX nomenclature committee has designated this allele as Triobp ${ }^{\text {tm }}$ ${ }_{1 \text { Sik }}$ (MGI:6189198) with an accession number at Riken of CDB0844K (http://www2.clst.riken.jp/arg/ mutant $\% 20$ mice $\% 20$ list.html). These mice are available to the scientific community.

Triobp ${ }^{4 E \times 8 / Y H B 226}$ compound heterozygous TRIOBP-5-deficient mice. A second TRIOBP-5 functional null mutant mouse was produced by crossing a Triobp $-4 / 5$ homozygous mutant mouse (Triobp ${ }^{4 E \times 8 / \Delta E \times 8}$ ) with a heterozygous Triobp-1/5 (Triobp ${ }^{\text {YHB226/+ }}$ ) mouse (Figure 1C). Individually, both of these alleles were reported

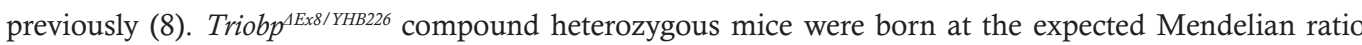
and were indistinguishable by weight and growth rate (data not shown) from their normal hearing heterozygous (Triobp ${ }^{4 E x /+}$ ) littermates. The contribution of TRIOBP-1 to stereocilia rootlet function, if any, awaits the development of a conditional knockout of TRIOBP-1 expression in hair cells, as a whole-body loss of TRIOBP-1 is lethal (8).

Genotyping and RT-PCR analysis. For Triobp $p^{4 E x-10 / 4 E x 9-10}$ mice, genotyping was performed with the following primers in a single PCR reaction: primer-P1 (WT.F), 5'-CCAAGGCTTCAGGACCAGAG-3'; primer-P2 (mut.F), 5'-CCTTCTATCGCCTTCTTGACGAG-3'; primer-P3 (rev), 5'-CACACTGCTTGTCAGCTGGT-3'. The product size of amplimers using primers P1/P3 (WT) and P2/P3 (KO) are $399 \mathrm{bp}$ and 276 bp, respectively. PCR conditions involved denaturation at $94^{\circ} \mathrm{C}$ for 2 minutes, $94^{\circ} \mathrm{C}$ for 30 seconds, $58^{\circ} \mathrm{C}$ for 30 seconds, and $68^{\circ} \mathrm{C}$ for 30 seconds, which was repeated for 35 cycles with a final extension at $68^{\circ} \mathrm{C}$ for 10 minutes. For R26-EGFP-Triobp-4 reporter mice, all primers were used in a single PCR reaction: primer-P1 (forward), 5'-TCCCTCGTGATCTGCAACTCCAGTC-3'; primer-P2 (WT.R), 5'-AACCCCAGATGACTACCTATCCTCC-3'; and primer-P3 (LoxP.R), 5'-GCTGCAGGTCGAGGGACC-3'; the product size of amplimers using primers $\mathrm{P} 1 / \mathrm{P} 2$ and $\mathrm{P} 1 / \mathrm{P} 3$ are $217 \mathrm{bp}$ and $270 \mathrm{bp}$, respectively. $\mathrm{PCR}$ reaction conditions involved a denaturation step at $96^{\circ} \mathrm{C}$ for 2 minutes, then $96^{\circ} \mathrm{C}$ for 30 seconds, $65^{\circ} \mathrm{C}$ for 30 seconds, and $72^{\circ} \mathrm{C}$ for 30 seconds, which was repeated 35 times, and a final extension at $72^{\circ} \mathrm{C}$ for 10 minutes.

Triobp ${ }^{4 E \times 8 / Y H B 226}$ compound heterozygous mice were genotyped using primers Tara Ex8.F3, 5'-CAGGCAACAATGTCTTGGCG-3'; Tara Ex8.WTR3, 5'-TGGGTAACGCCAGGGTTTTC-3'; and Tara Ex8. KOR1, 5'-GGTTCTGTTGAGAGAGGAGTCTTGG-3' as described previously (8), where the product sizes are $645 \mathrm{bp}$ for WT and $338 \mathrm{bp}$ for the mutant allele. The Triobp ${ }^{\text {YHB226 }}$ allele was genotyped using 3 primers in 1 tube: YHB226.F1, 5'-AGGCTCTCCGACTGCAATAA-3'; YHB226.En2ExRv, 5'-ATGCGATCTGCGTTCTTCTT-3'; and YHB226.WTR3, 5'-GCTTTTCCAACTCCTGCCACTTC-3'. The product size of the amplimer for WT is $424 \mathrm{bp}$ and $726 \mathrm{bp}$ for the mutant allele, using the same thermocycler conditions described previously (8). Briefly, after denaturation at $94^{\circ} \mathrm{C}$ for 2 minutes, there was 35 cycles of $94^{\circ} \mathrm{C}$ for 30 seconds, $55^{\circ} \mathrm{C}$ for 30 seconds, and $68^{\circ} \mathrm{C}$ for 1 minute and a final extension at $68^{\circ} \mathrm{C}$ for 10 minutes.

Internally deleted Triobp-5 transcripts were amplified from P3 WT cochlear cDNA using primers 
5'-TTCCAGACTCGGGATGAGGGAC-3' and 5'-CCCCTGTATCCCATTACTG-3' (8). Expression of this transcript in Triobp ${ }^{4 E \times 8 / Y H B 226}$ deaf heterozygotes was confirmed using a forward PCR primer that was complementary to the sequence at the junction of exons 14 and 15 (5'-CTTCCAGATCCACACCAAGGAC-3') and a reverse primer 5'-CCTGAAGAAGGTGCAAGGTAAAGAC-3' complementary to the sequence in exon 24. The products were verified by Sanger sequencing.

$A B R$ and DPOAE measurements. In each mouse, cochlear hearing function was tested by ABR and DPOAE at 4, 8, and 12 weeks of age inside a sound-proof booth (Acoustic Systems), while resting on a heating pad connected to a temperature controller and rectal probe (World Precision Instruments) to maintain body temperature near $37^{\circ} \mathrm{C}$. Mice were anesthetized via an intraperitoneal injection of a solution of ketamine $(56 \mathrm{mg} / \mathrm{kg})$ and Dexdomitor (Pfizer) $(0.375 \mathrm{mg} / \mathrm{kg})$. DPOAEs and ABRs were measured using Tucker-Davis Technologies (TDT) hardware (RZ6 Multi I/O processor, MF-1 speakers) and software (BioSigRz, v. 5.7 or 5.7.1). DPOAEs were recorded first using 2 TDT MF-1 speakers and an ER-10B+ microphone (Etymotic) coupled to the mouse's ear using a modified pipette tip. A $10-\mu 1$ pipette tip was trimmed to achieve $10 \mathrm{~mm}$ in length and $2 \mathrm{~mm}$ in the diameter at the tip opening. DPOAE $\left(2 f_{1}-f_{2}\right)$ levels were obtained in response to 2 primary tones at $f_{1}=65 \mathrm{~dB}$ sound pressure level (SPL) and $f_{2}=55 \mathrm{~dB}$ SPL, with $f_{2}$ varied from 4 to $44.8 \mathrm{kHz}$ (5 points/octave) and $f_{2} / f_{1}=1.25$. The mean noise floor was calculated from 3 points sampled above and 3 points sampled below the $2 f_{1}-f_{2}$ frequency. DPOAE signals were converted to $\mathrm{dB}$ SPL according to the calibration of an ER-10B+ microphone provided by the manufacturer. ABR thresholds were determined by presenting Blackman-gated tone-burst stimuli (3 ms duration, 29.9/s, alternating polarity) at 8,16 , and $32 \mathrm{kHz}$. Subdermal needle electrodes were placed at the cranial vertex and beneath each pinna, with the non-test ear serving as the ground. Stimuli were delivered via a TDT MF-1 speaker in a closed-field configuration. PVC tubing provided with the speaker was terminated in a modified pipette tip of the same dimensions as the one used with the DPOAE microphone. Responses were amplified $(20 \times)$, filtered $(0.3-3 \mathrm{kHz})$, and digitized at $25 \mathrm{kHz}$. Thresholds were determined through visual inspection of stacked waveforms (average of 512-1024 artifact-free responses per waveform). The stimulus was presented first at $80 \mathrm{~dB}$ SPL and then decreased in 10- to 20-dB steps until the ABR waveform disappeared. The stimulus was then increased and decreased in 5-dB steps until the lowest stimulus level that produced a repeatable waveform (ABR threshold) was determined. At least 2 waveforms $(1,024$ responses each) were obtained for stimulus levels at and near the ABR threshold to ensure repeatability of the response. If no response was evident at $80 \mathrm{~dB}$ SPL, the stimulus level was increased to $90 \mathrm{~dB}$ SPL and testing proceeded as described above. When no repeatable waves were visible at the highest stimulus level tested (90 dB SPL), the threshold was designated as $100 \mathrm{~dB}$ SPL for subsequent analyses.

$\beta$-Gal staining. Inner ears dissected from P13 Triobp $p^{\lfloor E x 9-10 /+}$ heterozygous mice were fixed with $0.2 \%$ weight per volume (w/v) glutaraldehyde and $2 \%(\mathrm{w} / \mathrm{v})$ paraformaldehyde (PFA) in PBS for 20 minutes at room temperature. The cochlear bone was removed in $2 \mathrm{mM} \mathrm{MgCl}_{2}, 0.01 \%$ sodium deoxycholate, and $0.02 \%$ Nonidet $\mathrm{P}-40$ in phosphate buffer ( $\mathrm{pH} 7.3$ ), and then stained with $2 \mathrm{mg} / \mathrm{ml}$ 5-bromo-4-chloro-3indolyl- $\beta$-D-galactoside (X-Gal), $15 \mathrm{mM}$ potassium ferricyanide, $15 \mathrm{mM}$ potassium ferrocyanide, $2 \mathrm{mM}$ $\mathrm{MgCl}_{2}, 0.01 \%$ sodium deoxycholate, and $0.02 \%$ Nonidet $\mathrm{P}-40$ in phosphate buffer ( $\mathrm{pH} 7.3$ ). After staining, samples were postfixed with $4 \%(\mathrm{w} / \mathrm{v})$ PFA in PBS at $4^{\circ} \mathrm{C}$ for 6 hours, incubated in $15 \%$ sucrose in PBS at $4^{\circ} \mathrm{C}$ overnight, mounted in OCT, and frozen in liquid nitrogen. Frozen sections were cut at $8-\mu \mathrm{m}$ thickness. WT P13 mouse cochleae were processed simultaneously to serve as a control. No $\beta$-Gal signal was observed in control inner ear tissues.

Gene gun-mediated transfection. Organ of Corti sensory epithelia were dissected from P0-P3 C57BL/6J WT mice and cultured overnight in DMEM/F12 medium supplemented with 7\% FBS at $37^{\circ} \mathrm{C}$ and $5 \% \mathrm{CO}_{2}$. Epithelial explants were then transfected with AcGFP1-Triobp-4 and AcGFP1-Triobp-5 using 1- $\mu \mathrm{m}$ gold carriers coated with cDNA and delivered via gene gun-mediated transfection using helium at 110 psi as described previously $(36,49)$. After 24 hours of transfection, sensory epithelial explants were washed 2 times in $1 \times$ PBS and fixed in 4\% PFA for 2 hours at room temperature or overnight at $4^{\circ} \mathrm{C}$, permeabilized in $0.1 \%$ Triton X-100, and counterstained with Rhodamine-phalloidin or Alexa Fluor 546-phalloidin at 1:100 dilution in 1× PBS for 15-20 minutes at room temperature following 3 washes of 10 minutes each in $1 \times$ PBS. Transfected and counterstained sensory epithelial explants were removed from the culture dish using a fine needle and mounted on a glass slide with Prolong Antifade (Thermo Fisher Scientific). The images were obtained using a Zeiss LSM780 confocal microscope equipped with a $63 \times, 1.4$ N.A. objective (Zeiss). 
Antibodies. The custom rabbit polyclonal antibodies against TRIOBP isoforms 4/5 and TRIOBP 5 were prepared and used as described previously (8). Mouse monoclonal anti-GFP antibody was obtained from Fujifilm Wako Pure Chemical Corporation (catalog 012-20461), and Alexa Fluor 488 goat anti-rabbit IgG pAb (catalog A11008), Alexa Fluor 546 goat anti-rat IgG pAb (catalog A11081), and Alexa Fluor 546-phalloidin (catalog A22283) were obtained from Thermo Fisher Scientific.

Immunofluorescence microscopy. The inner ears from mice at P5, P12, and P32 were dissected and fixed for 20 minutes with $2 \%(\mathrm{w} / \mathrm{v})$ formaldehyde in PBS at room temperature. Tissues were washed with $1 \times$ PBS, microdissected and permeabilized in $0.2 \%(\mathrm{w} / \mathrm{v})$ Triton X-100 for 15 minutes at room temperature. The samples were blocked with $2 \%$ bovine serum albumin (BSA) and $5 \%$ normal goat serum in PBS and incubated with primary antibodies at $5 \mu \mathrm{g} / \mathrm{ml}$ followed by Alexa Fluor-conjugated secondary antibodies at $2-5 \mu \mathrm{g} / \mathrm{ml}$. Samples were washed in $1 \times$ PBS 3 times, 10 minutes each, and mounted using ProLong Gold antifade reagent (Thermo Fisher Scientific) and imaged with an LSM780 and a 63× 1.4 NA objective. Some samples were mounted in Fluoromount-G (SouthernBiotech) and imaged with an SP8 confocal microscope (Leica Microsystems).

SEM. Inner ear tissue from mice at P7, P14, P30, and P40 was processed as described previously (50) or briefly fixed in 2\% (w/v) PFA in PBS followed by 2.5\% glutaraldehyde in $100 \mathrm{mM}$ HEPES buffer for 2 hours. After fixation, the bony capsule was decalcified with 5\% (w/v) EDTA for 24 hours in PBS, the organ of Corti was microdissected and dehydrated with a dilution series of ethanol $(50 \%, 60 \%, 70 \%, 80 \%, 90 \%$, $99 \%$, and $100 \%$ ), immersed in $t$-butanol, frozen at $-20^{\circ} \mathrm{C}$, and the $t$-butanol sublimated off. Samples were then sputter-coated with platinum-palladium alloy using an ion coater (IB-3; Eiko) and observed by SEM (S-4700 and S-4800, Hitachi).

TEM and serial section TEM. Cochleae were fixed in 2\% PFA and 2.5\% glutaraldehyde in HEPES, dehydrated in a series of ethanol dilutions $(65 \%, 75 \%, 85 \%, 95 \%, 99 \%$, and $100 \%$ ) followed by $100 \%$ propylene oxide and embedded in Epon 812. Ultrathin sections of 70-nm thickness were cut with a diamond knife and stained with uranyl acetate and/or lead citrate. Samples were observed by TEM (H-7650 and 1200, Hitachi or JEM-2100, JEOL).

For serial section TEM, whole P16 cochleae from WT and TRIOBP-mutant mice were thin-sectioned on a Leica Ultramicrotome at $60 \mathrm{~nm}$. Serial sections were placed on single-slot grids with a carbon/formvar film (EMS) and stained with lead citrate. Several series of sections ranging from 7-12 sections were examined in a JEM-2100 TEM (JEOL). For each section, the apex of the inner and outer hair cells from basal, middle, and apical turns were photographed at low, middle, and high magnification (total of 1,813 TEM micrographs). Subsequently, these images were rotated in Adobe Photoshop to adjust their orientation and processed using 3D-reconstruction software (Reconstruct, copyright 1996-2007 by John C. Fiala; funded by the Human Brain Project and NIH) (51).

FIB-SEM tomography. FIB-SEM samples were prepared by a modified Ellisman method (52). Samples were dissected as for TEM, and incubated with $1.5 \%$ potassium ferrocyanide followed by $2 \%$ osmium tetroxide in deionized water (DW) at $4^{\circ} \mathrm{C}$. After 1 hour, the tissues were washed with distilled water (DW) and fixed with $2 \%$ osmium tetroxide in DW at room temperature for 1 hour. The fixed cochleae were dehydrated with a dilution series of ethanol $(65 \%, 75 \%, 85 \%, 95 \%, 99 \%$, and $100 \%)$ and propylene oxide and were embedded in Epon 812.

The specimens were set on the stage of a FIB-SEM (Quanta 3D FEG, FEI or CrossBeam 540, Zeiss). Microfabrication around the reconstruction area was performed as previously described (53). Approximately 1,000 block-face serial images were acquired by repeated cycles of sample surface milling and imaging using Slice and View G2 software (FEI) and smartFIB (ZEISS), respectively. The milling was performed with a gallium ion beam at $30 \mathrm{kV}$ with a current of 2 or $7 \mathrm{nA}$. The milling pitch was set at $20 \mathrm{~nm} / \mathrm{step}$. The images were acquired at a landing energy of $2.5 \mathrm{keV}$ with a stage bias voltage of $3 \mathrm{kV}$ (Quanta FEI) or $1.5 \mathrm{keV}$ (CrossBeam Zeiss). The other acquisition parameters were as follows: beam current $=50 \mathrm{pA}$ or $1 \mathrm{nA}$, dwell time $=4 \mu \mathrm{s} /$ pixel, image size $=2048 \times 1766$ pixels $(10.0 \mu \mathrm{m} \times 10.9 \mu \mathrm{m})$, pixel size $=4.86 \mathrm{~nm} \times 6.17 \mathrm{~nm} /$ pixel; scan speed $=$ $2.8 \mathrm{~min} /$ image, image size $=4096 \times 3072$ pixels $(40.96 \mu \mathrm{m} \times 30.72 \mu \mathrm{m})$, pixel size $=10 \mathrm{~nm} \times 10 \mathrm{~nm} /$ pixel. The resultant image stacks were processed using Dragonfly 3.1 software (ORS Inc.) and Avizo 6.3 software (FEI).

Nanoscale pull-down (NanoSPD) protein-protein interaction assay. The possibility that TRIOBP-5 homomultimerizes was experimentally tested using NanoSPD 2.0 assays (42) and observations were evaluated using spatial correlation and intensity correlation analyses. A NanoSPD assay is an in vivo affinity pull down that harnesses the ability of the myosin-10 motor domain (heavy meromyosin, HMM) to move on 
F-actin to the tips of filopodia. Myosin-10 HMM was fused to the sequence of a GFP-Nanotrap (single-domain antibody) on the carboxy terminus of a myosin-10 HMM. The MYO10-HMM-nanotrap (Addgene, pcDNA3.1-MYO10-HMM-Nanotrap, 87255, abbreviated MYO10 NANOTRAP) binds to and transports a GFPtagged protein bait of interest to filopodia tips. Here, TRIOBP-5 as bait was epitope tagged with GFP, and when coexpressed in HeLa cells (ATCC, CCL-2) is transported to filopodia tips. At the same time, if HeLa cells are also transfected with DsRed-TRIOBP-5 as prey, and if TRIOBP-5 can homomultimerize, it is also transported to filopodia tips by the AcGFP1-TRIOBP-5 bound to the Nanotrap (42). The readout is yellow fluorescence at filopodia tips, indicating that an interaction between TRIOBP-5 molecules has occurred. Controls are shown in Figure 7 and Supplemental Figure 9). The Triobp-5 $5^{\Delta c c}$ expression vector in which the coiled-coil domains were deleted was constructed using 3 fragments from the full-length Triobp-5 cDNA that were combined using In-Fusion cloning (Takara, 638912), such that sequences encoded by exons 11, 12, and 15-22 were deleted. For transfection into HeLa cells in 6-well dishes, we used expression vectors (equal molar amounts for a total of $2.0 \mu \mathrm{g}$ of DNA) that separately express AcGFP1-TRIOBP-5, DsRed-TRIOBP-5, and the MYO10 ${ }^{\text {NANOTRAP }}$ per well. HeLa cell transfections were performed using Lipofectamine 3000 (Thermo Fisher Scientific, L3000008).

PFT-AFM imaging. Freshly dissected P5 organ of Corti sensory epithelium from Triobp ${ }^{4 E x 9-10 / \triangle E x 9-10}$ mutant and Triobp $p^{4 E x-10 /+}$ heterozygous littermates was plated on a glass-bottom dish (HBST-5040, Willco Wells) precoated with $10 \mu \mathrm{l}$ of Cell-Tak (Corning) and immersed in Leibovitz media (L-15, 21083-027, Life Technologies). Blinded to the genotype, PFT-AFM experiments were performed on a Bruker Bioscope Catalyst AFM system (Bruker) directly mounted on an inverted Axiovert 200M microscope (Zeiss) equipped with a confocal laser scanning microscope (510 Meta, Zeiss) and a 40× objective lens (0.95 NA, Plan-Apochromat, Zeiss). During experiments, explants were maintained at $37^{\circ} \mathrm{C}$ using a Bruker heated stage. An AFM probe specially designed for live cell imaging (PFQNM-LC, Bruker) has a tip height of 17 $\mu \mathrm{m}$, controlled tip radius of $65 \mathrm{~nm}$, and opening angle of $15^{\circ}$. The cantilevers (Bruker, PFQNM-LC-A$\mathrm{CAL}$ ) were precalibrated by the Bruker system using a laser Doppler vibrometer. The spring constant range of the cantilever was $0.06-0.08 \mathrm{~N} / \mathrm{m}$. For PFT-AFM imaging, we used a driving frequency of $500 \mathrm{~Hz}$ and drive amplitudes of $500 \mathrm{~nm}$ to $650 \mathrm{~nm}$. The scan speed used was $0.5 \mathrm{~Hz}$. The PeakForce feedback was set between $800 \mathrm{pN}$ and $1.2 \mathrm{nN}$. The scan resolution of all images was $256 \times 256$ pixels. The elastic Young's modulus (stiffness) calculations were performed using NanoScope Analysis software (Bruker). The elastic Young's modulus is a mechanical property that describes the stiffness of a material. It relates the stress against the strain of a material in the linear elastic region (43). The elastic Young's modulus ( $E$; Pa) was computed by fitting each force-distance curve with the Sneddon's contact mechanics model for indenting an infinite isotropic elastic half-space with a conical indenter:

$F_{\text {Sneddon }}=(8 E \tan \alpha / 3 \pi) \delta^{2}$, where $F$ is the applied force, $\alpha$ is the tip half-opening angle, and $\delta$ is the sample mean indentation.

Pivotal flexibility of hair cell stereocilia. Flexibility of the IHC bundles was estimated in acutely isolated mouse organ of Corti explants. The explants were dissected at P8 in L-15 cell medium and mounted in glass-bottom Petri dishes with a pair of dental floss monofilaments. IHC bundles were observed at room temperature with an upright microscope $(\mathrm{E} 600 \mathrm{FN}$, Nikon) equipped with a $60 \times$ water-immersion objective (1.0 NA, $2.0 \mathrm{~mm}$ working distance) and a 1.6× secondary magnification lens. IHC stereocilia bundles were deflected with a fluid-jet as described previously (8). Briefly, a micropipette of an approximately $6 \mu \mathrm{m}$ inner diameter and filled with bath solution (L-15, Invitrogen) was positioned at a distance of approximately 6 $\mu \mathrm{m}$ in front of the hair bundle and pressure steps were generated by a High-Speed Pressure Clamp (HSPC1, ALA Scientific) applied to the back of this pipette. According to our calibrations, the force generated by this microjet depends linearly on the applied pressure (Supplemental Figure 8 in ref. 8). The same pipette was used to deflect stereocilia bundles of WT and Triobp ${ }^{4 E x 9-10 / \Delta E x 9-10}$ mice. The steady-state pressure was adjusted to zero by monitoring debris movement in front of a fluid-jet.

Movements of stereocilia were video recorded with a CMOS camera (Flea3, PointGrey; FLIR Integrated Imaging Solutions) for subsequent frame-by-frame computation of displacements using algorithms developed for quantifying electromotility of isolated OHCs. Briefly, the intensity profiles across a middle of the bundle were collected for each frame of the video record. The numbers of points in these profiles were interpolated 50 times to obtain subpixel resolution. The frame-by-frame shifts of the intensity profiles, and hence bundle position, were determined by the least-squares algorithm. The typical error of the movement measurements with this technique is approximately $20 \mathrm{~nm}$ (54). Then, the slow drift of the bundle position, if present, was 
corrected, deflections of the bundle were automatically detected, and their amplitudes were measured with the drift correction and automated event detection features of the pClamp 10 software (Molecular Devices) that was developed for the analysis of single-channel activity in patch-clamp recordings.

Statistics. All experiments were repeated at least 3 times. Data are shown as mean \pm SEM or mean \pm SD as indicated in figure legends. Student's $t$ tests of independent variables (2-tailed distribution) or Mann-Whitney $U$ tests were used to determine statistical significance and a $P$ value less than 0.05 was considered significant.

Study approval. The use of mice was approved by the Animal Research Committee of Kyoto University Graduate School of Medicine (permit number 11179), the Animal Care and Use Committees at the $\mathrm{NIH}$ (protocol 1263 to TBF), the University of Kentucky Animal Care and Use Committee (protocol 903M2005 to GIF), and the Institutional Animal Care and Use Committee of RIKEN Kobe Branch (permit number A2001-03-68) and conducted according to the NIH Guide for Care and Use of Laboratory Animals (National Academies Press, 2011).

\section{Author contributions}

TK, IAB, TBF, and SK conceived the project and experiments. TK, IAB, AXCR, K. Ohta, SMC, RSP, K. Ono RT, AI, AR, HK, MK, YXW, TA, MI, CFF, GPR, EAW, TSF, KS, K. Omori, and JI conducted experiments. TK, IAB, GIF, TBF, and SK wrote the manuscript and all authors edited the manuscript.

\section{Acknowledgments}

This work was supported in part by NIH National Institute on Deafness and Other Communication Disorders (NIDCD) Intramural Research Programs (DC000039-20 to TBF, DC000080 to TSF, ZICDC000081 to RSP and Y-XW, and 1ZIADC00003322 to Richard Chadwick, who supports AXCR); by the Intramural Research Program of the National Institute of Mental Health/NIH (ZIAMH002946) who in part supports CFF; NIDCD/NIH (R01DC014658 to GIF), JSPS KAKENHI grant 26293369 and 17H04345 to SK; and JP16H06280 to K. Ota. We thank Joseph A. Duda, Barbara Zwiesler, Alec Callahan, Patrick Diers, Jim McGehee, and animal facility staff at NIDCD/NIH; and Benjamin Perrin and Douglas Epstein for critically reading the manuscript. We thank $\mathrm{H}$. Kohda and $\mathrm{K}$. Okamoto-Furuta for assistance with EM image acquisitions and Y. Kimura and Richard Chadwick for helpful discussions.

Address correspondence to: Thomas B. Friedman, National Institute on Deafness and Other Communication Disorders, NIH, 1F-141 Porter Neuroscience Center, 35 Convent Drive, Bethesda, Maryland 20892, USA. Phone 301.496.7882; Email: friedman@nidcd.nih.gov. Or to: Shin-ichiro Kitajiri, Department of Otorhinolaryngology, Shinshu University School of Medicine, 3-1-1 Asahi, Matsumoto, Nagano 3908621, Japan. Phone: 81.263.37.2666; Email: kitajiri@shinshu-u.ac.jp.

K. Ono's present address is: Laboratory of Molecular Biology, NIDCD, NIH, Bethesda, Maryland, USA.

AR's present address is: New York Genome Center, New York, New York, USA.

1. Wang H, et al. Novel variants identified in multiple sclerosis patients from southern China. Front Neurol. 2018;9:582

2. Bao J, Wang S, Gunther LK, Kitajiri S, Li C, Sakamoto T. The actin-bundling protein TRIOBP-4 and -5 promotes the motility of pancreatic cancer cells. Cancer Lett. 2015;356(2 Pt B):367-373.

3. Hoffmann TJ, Keats BJ, Yoshikawa N, Schaefer C, Risch N, Lustig LR. A large genome-wide association study of age-related hearing impairment using electronic health records. PLoS Genet. 2016;12(10):e1006371.

4. Pollak A, et al. Whole exome sequencing identifies TRIOBP pathogenic variants as a cause of post-lingual bilateral moderate-to-severe sensorineural hearing loss. BMC Med Genet. 2017;18(1):142.

5. Riazuddin S, et al. Mutations in TRIOBP, which encodes a putative cytoskeletal-organizing protein, are associated with nonsyndromic recessive deafness. Am J Hum Genet. 2006;78(1):137-143.

6. Shahin H, et al. Mutations in a novel isoform of TRIOBP that encodes a filamentous-actin binding protein are responsible for DFNB28 recessive nonsyndromic hearing loss. Am J Hum Genet. 2006;78(1):144-152.

7. Wesdorp M, et al. Broadening the phenotype of DFNB28: Mutations in TRIOBP are associated with moderate, stable hereditary hearing impairment. Hear Res. 2017;347:56-62.

8. Kitajiri S, et al. Actin-bundling protein TRIOBP forms resilient rootlets of hair cell stereocilia essential for hearing. Cell. 2010;141(5):786-798.

9. May V, Schiller MR, Eipper BA, Mains RE. Kalirin Dbl-homology guanine nucleotide exchange factor 1 domain initiates new axon outgrowths via RhoG-mediated mechanisms. J Neurosci. 2002;22(16):6980-6990.

10. Medley QG, Buchbinder EG, Tachibana K, Ngo H, Serra-Pagès C, Streuli M. Signaling between focal adhesion kinase and trio. 
J Biol Chem. 2003;278(15):13265-13270.

11. Peng YJ, et al. Trio is a key guanine nucleotide exchange factor coordinating regulation of the migration and morphogenesis of granule cells in the developing cerebellum. J Biol Chem. 2010;285(32):24834-24844.

12. Schmidt S, Debant A. Function and regulation of the Rho guanine nucleotide exchange factor Trio. Small GTPases. 2014;5:e29769.

13. Valdivia A, Goicoechea SM, Awadia S, Zinn A, Garcia-Mata R. Regulation of circular dorsal ruffles, macropinocytosis, and cell migration by RhoG and its exchange factor, Trio. Mol Biol Cell. 2017;28(13):1768-1781.

14. Bradshaw NJ, Yerabham ASK, Marreiros R, Zhang T, Nagel-Steger L, Korth C. An unpredicted aggregation-critical region of the actin-polymerizing protein TRIOBP-1/Tara, determined by elucidation of its domain structure. J Biol Chem. 2017;292(23):9583-9598.

15. Seipel K, O'Brien SP, Iannotti E, Medley QG, Streuli M. Tara, a novel F-actin binding protein, associates with the Trio guanine nucleotide exchange factor and regulates actin cytoskeletal organization. J Cell Sci. 2001;114(Pt 2):389-399.

16. Yano T, et al. Tara up-regulates E-cadherin transcription by binding to the Trio RhoGEF and inhibiting Rac signaling. $J$ Cell Biol. 2011;193(2):319-332.

17. Zhu Y, Wang C, Lan J, Yu J, Jin C, Huang H. Phosphorylation of Tara by Plk1 is essential for faithful chromosome segregation in mitosis. Exp Cell Res. 2012;318(18):2344-2352.

18. Bao J, et al. R1 motif is the major actin-binding domain of TRIOBP-4. Biochemistry. 2013;52(31):5256-5264.

19. Frolenkov GI, Belyantseva IA, Friedman TB, Griffith AJ. Genetic insights into the morphogenesis of inner ear hair cells. Nat Rev Genet. 2004;5(7):489-498.

20. Griffith AJ, Friedman TB. In: Wackym PA, Snow JB eds. Ballenger's Otolaryngology Head and Neck Surgery. Raileigh, NC: PMPH USA, Ltd; 2016:329-346.

21. Dallos P. Outer hair cells: the inside story. Ann Otol Rhinol Laryngol Suppl. 1997;168:16-22.

22. Ahmed ZM, et al. The tip-link antigen, a protein associated with the transduction complex of sensory hair cells, is protocadherin-15. J Neurosci. 2006;26(26):7022-7034.

23. Corey DP, Holt JR. Are TMCs the mechanotransduction channels of vertebrate hair cells? J Neurosci. 2016;36(43):10921-10926

24. Cunningham CL, Müller U. Molecular structure of the hair cell mechanoelectrical transduction complex. Cold Spring Harb Perspect Med. 2019;9(5):a033167.

25. Kurima K, et al. TMC1 and TMC2 localize at the site of mechanotransduction in mammalian inner ear hair cell stereocilia. Cell Rep. 2015;12(10):1606-1617.

26. Furness DN, Mahendrasingam S, Ohashi M, Fettiplace R, Hackney CM. The dimensions and composition of stereociliary rootlets in mammalian cochlear hair cells: comparison between high- and low-frequency cells and evidence for a connection to the lateral membrane. J Neurosci. 2008;28(25):6342-6353.

27. Itoh M. Preservation and visualization of actin-containing filaments in the apical zone of cochlear sensory cells. Hear Res. 1982;6(3):277-289.

28. Kimura RS. The ultrastructure of the organ of Corti. Int Rev Cytol. 1975;42:173-222.

29. Furness DN, Katori Y, Mahendrasingam S, Hackney CM. Differential distribution of beta- and gamma-actin in guinea-pig cochlear sensory and supporting cells. Hear Res. 2005;207(1-2):22-34.

30. Tilney LG, DeRosier DJ. Actin filaments, stereocilia, and hair cells of the bird cochlea. IV. How the actin filaments become organized in developing stereocilia and in the cuticular plate. Dev Biol. 1986;116(1):119-129.

31. Alford BR, Ruben RJ. Physiological, behavioral and anatomical correlates of the development of hearing in the mouse. Ann Otol Rhinol Laryngol. 1963;72:237-247.

32. Ehret G. Development of absolute auditory thresholds in the house mouse (Mus musculus). J Am Audiol Soc. 1976;1(5):179-184.

33. Mikaelian D, Alford BR, Ruben RJ. Cochlear potentials and 8 nerve action potentials in normal and genetically deaf mice. Ann Otol Rhinol Laryngol. 1965;74:146-157.

34. Nam JH, Fettiplace R. Force transmission in the organ of Corti micromachine. Biophys J. 2010;98(12):2813-2821.

35. Zhao HB, Santos-Sacchi J. Effect of membrane tension on gap junctional conductance of supporting cells in Corti's organ. $J$ Gen Physiol. 1998;112(4):447-455.

36. Belyantseva IA. Helios( $\left.{ }^{\circledR}\right)$ gene gun-mediated transfection of the inner ear sensory epithelium: recent updates. Methods Mol Biol. 2016;1427:3-26.

37. Davis H. An active process in cochlear mechanics. Hear Res. 1983;9(1):79-90.

38. Liberman MC, Dodds LW. Acute ultrastructural changes in acoustic trauma: serial-section reconstruction of stereocilia and cuticular plates. Hear Res. 1987;26(1):45-64.

39. Slepecky N, Hamernik R, Henderson D, Coling D. Correlation of audiometric data with changes in cochlear hair cell stereocilia resulting from impulse noise trauma. Acta Otolaryngol. 1982;93(5-6):329-340.

40. Liberman MC. Chronic ultrastructural changes in acoustic trauma: serial-section reconstruction of stereocilia and cuticular plates. Hear Res. 1987;26(1):65-88.

41. Lupas A, Van Dyke M, Stock J. Predicting coiled coils from protein sequences. Science. 1991;252(5009):1162-1164.

42. Bird JE, et al. Harnessing molecular motors for nanoscale pulldown in live cells. Mol Biol Cell. 2017;28(3):463-475.

43. Hibbeler RC. Mechanics of Materials. London, UK:Pearson; 2011:83.

44. Ren T, He W, Kemp D. Reticular lamina and basilar membrane vibrations in living mouse cochleae. Proc Natl Acad Sci USA. 2016;113(35):9910-9915.

45. Szarama KB, Gavara N, Petralia RS, Kelley MW, Chadwick RS. Cytoskeletal changes in actin and microtubules underlie the developing surface mechanical properties of sensory and supporting cells in the mouse cochlea. Development. 2012;139(12):2187-2197.

46. Abe T, et al. Establishment of conditional reporter mouse lines at ROSA26 locus for live cell imaging. Genesis. 2011;49(7):579-590.

47. Ikeya M, et al. Gene disruption/knock-in analysis of mONT3: vector construction by employing both in vivo and in vitro recombinations. Int J Dev Biol. 2005;49(7):807-823.

48. Yagi T, et al. A novel ES cell line, TT2, with high germline-differentiating potency. Anal Biochem. 1993;214(1):70-76. 
49. Belyantseva IA, Boger ET, Friedman TB. Myosin XVa localizes to the tips of inner ear sensory cell stereocilia and is essential for staircase formation of the hair bundle. Proc Natl Acad Sci USA. 2003;100(24):13958-13963.

50. Morozko EL, et al. ILDR1 null mice, a model of human deafness DFNB42, show structural aberrations of tricellular tight junctions and degeneration of auditory hair cells. Hum Mol Genet. 2015;24(3):609-624.

51. Fiala JC. Reconstruct: a free editor for serial section microscopy. J Microsc. 2005;218(Pt 1):52-61.

52. West JB, Fu Z, Deerinck TJ, Mackey MR, Obayashi JT, Ellisman MH. Structure-function studies of blood and air capillaries in chicken lung using 3D electron microscopy. Respir Physiol Neurobiol. 2010;170(2):202-209.

53. Ohta K, Sadayama S, Togo A, Higashi R, Tanoue R, Nakamura K. Beam deceleration for block-face scanning electron microscopy of embedded biological tissue. Micron. 2012;43(5):612-620.

54. Frolenkov GI, Kalinec F, Tavartkiladze GA, Kachar B. Cochlear outer hair cell bending in an external electric field. Biophys J. 1997;73(3):1665-1672. 